\title{
Warped Convolutions, Rieffel Deformations and the Construction of Quantum Field Theories
}

\author{
Detlev Buchholz ${ }^{1, *}$, Gandalf Lechner ${ }^{2}$, Stephen J. Summers ${ }^{3, * *}$ \\ 1 Institut für Theoretische Physik and Courant Centre "Higher Order Structures in Mathematics", \\ Universität Göttingen, 37077 Göttingen, Germany. \\ E-mail: buchholz@Theorie.Physik.UNI-Goettingen.DE \\ 2 Fakultät für Physik, Universität Wien, 1090 Vienna, Austria. E-mail: gandalf.lechner@univie.ac.at \\ 3 Department of Mathematics, University of Florida, Gainesville, FL 32611, USA. E-mail: sjs@ufl.edu
}

Received: 15 May 2010 / Accepted: 24 May 2010

Published online: 9 October 2010 - (C) The Author(s) 2010. This article is published with open access at Springerlink.com

\section{Dedicated to Sergio Doplicher on the occasion of his seventieth birthday}

\begin{abstract}
Warped convolutions of operators were recently introduced in the algebraic framework of quantum physics as a new constructive tool. It is shown here that these convolutions provide isometric representations of Rieffel's strict deformations of $C^{*}-$ dynamical systems with automorphic actions of $\mathbb{R}^{n}$, whenever the latter are presented in a covariant representation. Moreover, the device can be used for the deformation of relativistic quantum field theories by adjusting the convolutions to the geometry of Minkowski space. The resulting deformed theories still comply with pertinent physical principles and their Tomita-Takesaki modular data coincide with those of the undeformed theory; but they are in general inequivalent to the undeformed theory and exhibit different physical interpretations.
\end{abstract}

\section{Introduction}

Recent advances in algebraic quantum field theory have led to purely algebraic constructions of quantum field models on Minkowski space, both classical and noncommutative $[5,9,11-13,17,18,22-24,26,29]$, many of which cannot be achieved by the standard methods of constructive quantum field theory. Some of these models are local and free, some are local and have nontrivial $S$-matrices, and yet others manifest only certain remnants of locality, though these remnants suffice to enable the computation of nontrivial $S$-matrix elements.

In order to construct a quantum field model on noncommutative Minkowski space, Grosse and one of us [17] have deformed the free quantum field in a certain manner to find a family of theories which are Poincaré covariant and comply with a slightly weakened version of the principle of Einstein causality ("wedge locality"). As pointed out in [17], a completely analogous deformation can be carried out on a free field on

\footnotetext{
* Supported by the German Research Foundation (Deutsche Forschungsgemeinschaft (DFG)) through the Institutional Strategy of the University of Göttingen.

** Research supported by the NSF Grant DMS-0901370.
} 
classical Minkowski space. In [13] two of us presented a generalization (called a warped convolution) of that deformation which can be applied to any Minkowski space quantum field model in any number of dimensions. This deformation results in a family of distinct theories which are wedge-local and covariant under the representation of the Poincare group associated with the initial, undeformed theory. It turns out that also the $S$-matrix changes under this deformation, and the scattering is nontrivial even if the scattering of the initial theory is trivial. When taking the free quantum field as the initial model, this deformation coincides with that of Grosse and Lechner. It provides the first fully consistent examples of relativistic quantum field theories on four-dimensional Minkowski space describing nontrivial elastic scattering processes $[13,17]$.

Warped convolution was subsequently studied in the language of Wightman quantum field theory in [18], where it was shown that the deformation of the field operators can be understood as resulting in a certain deformation of the canonical product on the Borchers-Uhlmann algebra - the algebra of test functions canonically associated with a Wightman theory. This was the first indication that the deformation of operators resulting from warped convolution may be equivalent to a deformation of the operator product.

A well known example of this latter type is the strict deformation theory of $C^{*}-$ dynamical systems with an action of $\mathbb{R}^{n}$ developed by Rieffel in [28]. It was originally introduced for the quantization of classical models. We shall show in this paper that the warped convolution applied to any $C^{*}$-dynamical system provides a covariant representation of the corresponding deformed Rieffel algebra. In particular, all states in a covariant representation of the initial system can be lifted to states on the deformed algebra (compare [21, Cor. 4.4]).

In spite of this tight relation between the two deformation procedures, the concept of warped convolution appears to be more appropriate in applications to quantum field theory. For there one has to deal simultaneously with a multitude of different deformations and to establish relations between the resulting operators. This can be done most conveniently in a common representation space of the various Rieffel algebras, and such a space is provided by the warped convolution procedure. Suitably adjusting the deformation parameters to the geometry of Minkowski space, we apply the warped convolutions to quantum field theories, as outlined in [13], and prove as well as extend the results given there. As we shall further explain, any quantum field theory on Minkowski space can be constructed from a (causal) Borchers triple consisting of a von Neumann algebra, a representation of the Poincaré group and a vector representing the vacuum state. The physical constraints of causality and covariance can conveniently be expressed in terms of a few conditions on these triples. We shall show that these properties are preserved under a distinguished group of warped convolutions, thereby giving rise to interesting new theories.

The article is organized as follows. In Sect. 2 we prove and extend the results about warped convolution given in [13]. These extensions allow us to establish the relation with Rieffel deformed dynamical systems. In Sect. 3 a restricted family of warped convolutions is applied to Borchers triples to construct quantum field theories in two spacetime dimensions. We show, in particular, that the Tomita-Takesaki modular objects associated with such triples remain fixed under these deformations. The application of the warped convolutions to general relativistic quantum field theories in higher dimensions is discussed in Sect. 4. We present there the salient results given in [13] in the framework of causal Borchers triples and also establish further physically relevant properties of the deformed theories, not addressed in [13]. Finally, we briefly discuss prospects for further development of this approach in Sect. 5. 


\section{Warped Convolutions and Rieffel Deformations}

We clarify here the relation between the notion of warped convolution, recently introduced in [13], and the strict deformation of $C^{*}$-algebras established in [28] by Rieffel. In either case one proceeds from a $C^{*}$-dynamical system $\left(\mathcal{A}, \mathbb{R}^{n}\right), c f$. [27]. It consists of a $C^{*}$-algebra $\mathcal{A}$ equipped with a strongly continuous automorphic action of the group $\mathbb{R}^{n}$ which will be denoted by $\alpha$.

In order to relate the two settings, it will be convenient to consider the system $\left(\mathcal{A}, \mathbb{R}^{n}\right)$ in a covariant representation. That is, we regard $\mathcal{A}$ as a concrete $C^{*}$-algebra on a Hilbert space $\mathcal{H}$ on which the automorphisms $\alpha$ are implemented by the adjoint action of a weakly continuous unitary representation $U$ of $\mathbb{R}^{n}$,

$$
\alpha_{x}(A)=U(x) A U(x)^{-1}, \quad x \in \mathbb{R}^{n} .
$$

As a matter of fact, this assumption imposes no significant restriction of generality. For if the abstract algebra $\mathcal{A}$ can be represented faithfully on some separable Hilbert space, then there also exists a faithful covariant representation of $\left(\mathcal{A}, \mathbb{R}^{n}\right), c f$. [27, Lemma 7.4.9 and Prop. 7.4.7]. Furthermore, since the adjoint action $\alpha$ of the unitary representation $U$ can be extended to the algebra $\mathcal{B}(\mathcal{H})$ of all bounded operators on $\mathcal{H}$, no generality will be lost when we proceed to the $C^{*}$-dynamical system $\left(\mathcal{C}, \mathbb{R}^{n}\right)$, where $\mathcal{C} \subset \mathcal{B}(\mathcal{H})$ is the $C^{*}$-algebra of all operators on which $\alpha$ acts strongly continuously. We shall then be able to restrict to suitable subalgebras $\mathcal{A}$ of $\mathcal{C}$ as necessary.

2.1. Rieffel deformations. We begin by considering the $C^{*}$-algebra $\mathcal{C}$ of all uniformly continuous bounded functions $\boldsymbol{A}: \mathbb{R}^{n} \rightarrow \mathcal{B}(\mathcal{H})$. The algebraic structure of $\mathcal{C}$ is the natural one inherited from $\mathcal{B}(\mathcal{H})$, i.e. the algebraic operations in $\mathcal{C}$ are pointwise defined,

$$
(\boldsymbol{A}+\boldsymbol{B})(x)=\boldsymbol{A}(x)+\boldsymbol{B}(x),(\boldsymbol{A} \boldsymbol{B})(x)=\boldsymbol{A}(x) \boldsymbol{B}(x), \boldsymbol{A}^{*}(x)=\boldsymbol{A}(x)^{*} \quad x \in \mathbb{R}^{n},
$$

and the norm is given by ${ }^{1}$

$$
\|\boldsymbol{A}\|=\sup _{x \in \mathbb{R}^{n}}\|\boldsymbol{A}(x)\| .
$$

Following Rieffel [28], we consider the subalgebra $\mathcal{C}^{\infty} \subset \mathcal{C}$ of smooth (in the norm topology) elements $\boldsymbol{A}$, i.e. $\left\|\partial^{\mu} \boldsymbol{A}\right\|<\infty$ for all multi-indices $\mu{ }^{2}$ Note that the elements $C \in \mathcal{B}(\mathcal{H})$ act as multipliers on $\mathcal{C}^{\infty}$, if one identifies $C$ with the corresponding constant function in $\mathcal{C}^{\infty}$ (denoted by the same symbol). Clearly, the maps $(C, A) \mapsto C A$ and $(C, A) \mapsto A C$ are norm continuous in both variables, $\|C A\| \leq\|C\|\|\boldsymbol{A}\| \geq\|\boldsymbol{A C}\|$, and the multiplication by $C$ commutes with the operations of differentiation and integration on $\mathcal{C}^{\infty}$.

In the subsequent analysis we shall find it necessary to integrate the functions $x \mapsto$ $\boldsymbol{A}(x)$. In order to handle the fact that these functions are in general not absolutely integrable with respect to Lebesgue measure due to a lack of suitable decay properties, we introduce mollifiers $L_{n}: \mathbb{R}^{n} \rightarrow \mathbb{C}$. A convenient choice is given by

$$
L_{n}(x)=\left(i+x_{1}+\cdots+x_{n}\right)^{-1} \prod_{k=1, \ldots, n}\left(i+x_{k}\right)^{-1}, \quad x \in \mathbb{R}^{n} .
$$

\footnotetext{
${ }^{1}$ Risking some confusion, we use the same symbol for the norms on $\mathcal{C}$ and $\mathcal{B}(\mathcal{H})$.

${ }^{2}$ We use the notation $\mu=\left(\mu_{1}, \ldots, \mu_{n}\right), \partial_{x}^{\mu}=\partial_{x_{1}}^{\mu_{1}} \ldots \partial_{x_{n}}^{\mu_{n}}$ and $\left(\partial^{\mu} \boldsymbol{A}\right)(x)=\partial_{x}^{\mu} \boldsymbol{A}(x)$, where $x_{k}$ are the components of $x$ with respect to a fixed orthonormal basis in $\mathbb{R}^{n}$ and $\partial_{x_{k}}$ are the corresponding partial derivatives, $k=1, \ldots n$.
} 
Because of this simple form one easily verifies that $\left(\partial^{\mu} L_{n}\right)(x)=N_{n, \mu}(x) L_{n}(x)$, where $N_{n, \mu}$ is smooth and bounded for any multi-index $\mu$; moreover $L_{n} \in L^{1}\left(\mathbb{R}^{n}\right)$. We therefore choose $L_{n}$ as a universal mollifier on $\mathcal{C}^{\infty}$.

It follows from the preceding remarks that, for any multi-index $\mu$, the functions $x \mapsto \partial_{x}^{\mu}\left(L_{n}(x) \boldsymbol{A}(x)\right)$ are Bochner integrable in $\mathcal{B}(\mathcal{H})$ with respect to the Lebesgue measure. Moreover, applying Leibniz's rule, one gets

$$
\int d x\left\|\partial_{x}^{\mu}\left(L_{n}(x) A(x)\right)\right\| \leq c_{n, \mu}\|\boldsymbol{A}\|_{|\mu|}, \quad \boldsymbol{A} \in \mathcal{C}^{\infty},
$$

where $c_{n, \mu}$ does not depend on $\boldsymbol{A}$, and we have introduced the norms, $m \in \mathbb{N}_{0}$,

$$
\|\boldsymbol{A}\|_{m}=\sum_{\mu,|\mu| \leq m}\left\|\partial^{\mu} \boldsymbol{A}\right\| .
$$

The following technical lemma is a basic ingredient in the subsequent discussion. In its proof, we make use of arguments furnished by Rieffel [28].

Lemma 2.1. Let $\boldsymbol{A}, \boldsymbol{B} \in \mathcal{C}$ be $n+1$ times continuously differentiable and let $f \in$ $\mathcal{S}\left(\mathbb{R}^{n} \times \mathbb{R}^{n}\right)$ with $f(0,0)=1$.

(i) The norm limit of Bochner integrals in $\mathcal{B}(\mathcal{H})$,

$$
\lim _{\varepsilon \rightarrow 0}(2 \pi)^{-n} \iint d x d y f(\varepsilon x, \varepsilon y) e^{-i x y} \boldsymbol{A}(x) \boldsymbol{B}(y) \doteq \boldsymbol{A} \times \boldsymbol{B},
$$

exists and does not depend on $f$. Here $x y, x, y \in \mathbb{R}^{n}$ is any symmetric bilinear form on $\mathbb{R}^{n}$ with determinant 1 or -1 .

(ii) With $L_{n}$ as above, there exists a polynomial $u, v \mapsto P_{n}(u, v)$ on $\mathbb{R}^{n} \times \mathbb{R}^{n}$ of degree $n+1$ in the components of $u$ and $v$, respectively, such that

$$
\boldsymbol{A} \times \boldsymbol{B}=(2 \pi)^{-n} \iint d x d y e^{-i x y} P_{n}\left(\partial_{x}, \partial_{y}\right) L_{n}(x) \boldsymbol{A}(x) L_{n}(y) \boldsymbol{B}(y),
$$

where the integral is defined as a Bochner integral in $\mathcal{B}(\mathcal{H})$.

(iii) $\|\boldsymbol{A} \times \boldsymbol{B}\| \leq c_{n}\|\boldsymbol{A}\|_{n+1}\|\boldsymbol{B}\|_{n+1}$, for a universal constant $c_{n}$.

(iv) Let $C \in \mathcal{B}(\mathcal{H})$. Then

$$
\begin{aligned}
(C \boldsymbol{A} \times \boldsymbol{B}) & =C(\boldsymbol{A} \times \boldsymbol{B}), \quad(\boldsymbol{A} \times \boldsymbol{B} C)=(\boldsymbol{A} \times \boldsymbol{B}) C, \\
(\boldsymbol{A C} \times \boldsymbol{B}) & =(\boldsymbol{A} \times C \boldsymbol{B}),
\end{aligned}
$$

and the linear map

$$
C \mapsto \boldsymbol{A} \times C \boldsymbol{B}
$$

is continuous on the unit sphere of $\mathcal{B}(\mathcal{H})$ in the strong operator topology.

Proof. (i) Crucial for the result are certain properties of the function $x, y \mapsto e^{-i x y}$. Namely, for each polynomial $x, y \mapsto Q_{n}(x, y)$ of degree $n+1$ in the variables $x$ and $y$, respectively, there is a corresponding polynomial $P_{n}$ in the same family such that

$$
Q_{n}(x, y) e^{-i x y}=P_{n}\left(-\partial_{x},-\partial_{y}\right) e^{-i x y},
$$


and vice versa. The step from the right-hand side to the left-hand side is easily accomplished by differentiation; the opposite direction can likewise be established, noticing that the Fourier transform of $x, y \mapsto e^{-i x y}$ is again of this form. Choosing

$$
Q_{n}(x, y)=L_{n}(x)^{-1} L_{n}(y)^{-1}, \quad x, y \in \mathbb{R}^{n},
$$

with $L_{n}$ specified above, one observes that

$$
\begin{aligned}
& \iint d x d y f(\varepsilon x, \varepsilon y) e^{-i x y} \boldsymbol{A}(x) \boldsymbol{B}(y) \\
& =\iint d x d y Q_{n}(x, y) e^{-i x y} f(\varepsilon x, \varepsilon y) L_{n}(x) \boldsymbol{A}(x) L_{n}(y) \boldsymbol{B}(y) \\
& \left.=\iint d x d y\left(P_{n}\left(-\partial_{x},-\partial_{y}\right)\right) e^{-i x y}\right) f(\varepsilon x, \varepsilon y) L_{n}(x) \boldsymbol{A}(x) L_{n}(y) \boldsymbol{B}(y) \\
& =\iint d x d y e^{-i x y} P_{n}\left(\partial_{x}, \partial_{y}\right) f(\varepsilon x, \varepsilon y) L_{n}(x) \boldsymbol{A}(x) L_{n}(y) \boldsymbol{B}(y),
\end{aligned}
$$

where $P_{n}$ is the polynomial corresponding to the chosen $Q_{n}$. In view of the smoothness and rapid decay properties of the integrands, the integrals are defined as Bochner integrals in $\mathcal{B}(\mathcal{H})$, and the last equality is obtained by partial integration. Decomposing $P_{n}\left(\partial_{x}, \partial_{y}\right)$ into a sum of monomials of the form $\partial_{x}^{\mu} \partial_{y}^{v}$ with $|\mu|,|v| \leq n+1$, performing the differentiations and taking into account the properties of $L_{n}$, one gets by an application of the dominated convergence theorem,

$$
\begin{aligned}
& \lim _{\varepsilon \rightarrow 0} \iint d x d y e^{-i x y} P_{n}\left(\partial_{x}, \partial_{y}\right) f(\varepsilon x, \varepsilon y) L_{n}(x) \boldsymbol{A}(x) L_{n}(y) \boldsymbol{B}(y) \\
& \quad=\iint d x d y e^{-i x y} P_{n}\left(\partial_{x}, \partial_{y}\right) L_{n}(x) \boldsymbol{A}(x) L_{n}(y) \boldsymbol{B}(y)
\end{aligned}
$$

Note that the derivatives of $x, y \mapsto f(\varepsilon x, \varepsilon y)$ contain powers of $\varepsilon$ as factors and therefore disappear in the limit. In particular, the limit does not depend on the choice of $f$.

Assertion (ii) has been established in (2.1). From this relation one also obtains the estimate (iii), because of the properties of $L_{n}$ established above.

The proof of the equalities in (iv) is another straightforward consequence of (ii) and is therefore omitted. It remains to establish the continuity of the map. In view of the continuity and decay properties of the functions appearing in the representation (ii) of the product $\times$, the integrals underlying the definition of $\boldsymbol{A} \times \boldsymbol{C} \boldsymbol{B}$ can be approximated in norm, uniformly for $C \in \mathcal{B}(\mathcal{H}),\|C\| \leq 1$, by finite sums of the form

$$
\sum_{\mu, v, i, k} c_{\mu, v, i, k}\left(\partial^{\mu} \boldsymbol{A}\right)\left(x_{i}\right) C\left(\partial^{v} \boldsymbol{B}\right)\left(y_{k}\right),
$$

where $c_{\mu . v, i, k}$ are constants which do not depend on $C$. Since the operators $\left(\partial^{\mu} \boldsymbol{A}\right)\left(x_{i}\right)$, $\left(\partial^{v} \boldsymbol{B}\right)\left(y_{k}\right)$ are bounded, the stated continuity properties of the map with respect to $C$ then follow.

Within this general setting, the Rieffel deformations of the $C^{*}$-dynamical system $\left(\mathcal{C}, \mathbb{R}^{n}\right)$ [28] can be presented as follows. Let $\mathcal{C}^{\infty} \subset \mathcal{C}$ be the ${ }^{*}$-algebra of smooth elements with respect to the action of $\alpha$ and let $Q$ be a real skew symmetric matrix 
relative to the chosen bilinear form on $\mathbb{R}^{n}$, i.e. $x Q y=-y Q x, x, y \in \mathbb{R}^{n}$. One then considers for $A, B \in \mathcal{C}^{\infty}$ the functions in $\mathcal{C}^{\infty}$ given by

$$
x \mapsto \boldsymbol{A}_{\boldsymbol{\alpha}_{Q}}(x) \doteq \alpha_{Q x}(A), \quad y \mapsto \boldsymbol{B}_{\boldsymbol{\alpha}}(y) \doteq \alpha_{y}(B)
$$

and sets

$$
A \times{ }_{Q} B \doteq \boldsymbol{A}_{\alpha_{Q}} \times \boldsymbol{B}_{\boldsymbol{\alpha}}, \quad A, B \in \mathcal{C}^{\infty} .
$$

It has been shown by Rieffel [28] that $\times_{Q}$ defines an associative product on $\mathcal{C}^{\infty}$, the Rieffel product, which is compatible with the ${ }^{*}$-operation. In view of the normalization of the bilinear form chosen in Lemma 2.1 (i), the original identity operator 1 still acts as the identity with respect to the new product $\times_{Q}$. Moreover, there exists a $C^{*}-$ norm on the deformed algebra $\left(\mathcal{C}^{\infty}, \times_{Q}\right)$. It is of interest here that, by Lemma 2.1 , the Rieffel product extends to more general functions in $\mathcal{C}$ in a natural manner. We shall take advantage of this fact in the following subsection.

2.2. Warped convolution. We turn now to the discussion of the warped convolution on $\left(\mathcal{C}, \mathbb{R}^{n}\right)$ introduced in [13]. Many of the results below were stated there and provided with sketches of proofs; in addition to supplying complete proofs of those assertions, here we also prove results which strengthen and complement those discussed in [13].

The weakly continuous unitary representation $U$ of $\mathbb{R}^{n}$ implementing $\alpha$ enters into the definition of the warped convolution. In a first step we want to give proper meaning to the formal expressions $\int_{B} \alpha_{Q x}(A) d E(x)$ and $\int_{B} d E(x) \alpha_{Q x}(A), A \in \mathcal{C}^{\infty}$, where $Q$ is any real $n \times n$ matrix, $E$ is the spectral resolution of $U$ and $B \subset \mathbb{R}^{n}$ is any bounded Borel set. If $F$ is a finite-dimensional projection, it follows from the spectral calculus that the integrals $\int_{B} \alpha_{Q x}(A) F d E(x)$ and $\int_{B} d E(x) F \alpha_{Q x}(A)$ are well-defined in the strong operator topology. Moreover, since $U(y)=\int e^{i x y} d E(x), y \in \mathbb{R}^{n}$, one obtains for any test function $f$ as in Lemma 2.1,

$$
\begin{aligned}
& \int_{B} \alpha_{Q x}(A) F d E(x)=\lim _{\varepsilon \rightarrow 0}(2 \pi)^{-n} \iint d x d y f(\varepsilon x, \varepsilon y) e^{-i x y} \alpha_{Q x}(A) F E(B) U(y), \\
& \int_{B} d E(x) F \alpha_{Q x}(A)=\lim _{\varepsilon \rightarrow 0}(2 \pi)^{-n} \iint d x d y f(\varepsilon x, \varepsilon y) e^{-i x y} E(B) U(x) F \alpha_{Q y}(A) .
\end{aligned}
$$

Hence, adopting the notation in Lemma 2.1,

$$
\int_{B} \alpha_{Q x}(A) F d E(x)=\boldsymbol{A}_{\boldsymbol{\alpha}_{Q}} \times F \boldsymbol{U}_{\boldsymbol{B}}, \quad \int_{B} d E(x) F \alpha_{Q x}(A)=\boldsymbol{U}_{\boldsymbol{B}} F \times \boldsymbol{A}_{\boldsymbol{\alpha}_{Q}},
$$

where we have introduced the function $x \mapsto \boldsymbol{U}_{\boldsymbol{B}}(x) \doteq E(B) U(x)$, which is an element of $\mathcal{C}^{\infty}$ since the set $B$ is bounded. Choosing any net of projections $F$ converging monotonically to the identity operator 1, it follows from part (iv) of Lemma 2.1 that the right-hand side of these equalities converges in the strong operator topology. Hence the corresponding limits of the integrals on the left-hand side exist in $\mathcal{B}(\mathcal{H})$ and can be used to define the warped convolution integrals as

$$
\begin{aligned}
\int_{B} \alpha_{Q x}(A) d E(x) & \doteq \lim _{F \nearrow 1} \int_{B} \alpha_{Q x}(A) F d E(x)=\boldsymbol{A}_{\boldsymbol{\alpha}_{Q}} \times \boldsymbol{U}_{\boldsymbol{B}}, \\
\int_{B} d E(x) \alpha_{Q x}(A) & \doteq \lim _{F \nearrow 1} \int_{B} d E(x) F \alpha_{Q x}(A)=\boldsymbol{U}_{\boldsymbol{B}} \times \boldsymbol{A}_{\boldsymbol{\alpha}_{Q}} .
\end{aligned}
$$


If the spectrum $\operatorname{sp} U$ of $U$ is compact, the integrals do not depend on $B$ if $B \supset \operatorname{sp} U$, so one can proceed to the limit $B \nearrow \mathbb{R}^{n}$ in $\mathcal{B}(\mathcal{H})$. In the general case, however, we have a priori no control on the continuity properties of the resulting operators. In order to cope with this problem, we consider the dense domain $\mathcal{D} \subset \mathcal{H}$ of vectors which are smooth with respect to the action of $U$. Let $P=\left(P_{1}, \ldots, P_{n}\right)$ be the generators of $U$ and let

$$
x \mapsto\left(\boldsymbol{U}_{\mathbb{R}^{n}}\left(1+P^{2}\right)^{-n-1}\right)(x) \doteq U(x)\left(1+P^{2}\right)^{-n-1} .
$$

This function is an element of $\mathcal{C}$ which is $n+1$ times continuously differentiable. Making use of the first half of part (iv) of Lemma 2.1, we thus get for $\Phi \in \mathcal{D}$,

$$
\begin{aligned}
\left(\boldsymbol{A}_{\boldsymbol{\alpha}_{Q}} \times \boldsymbol{U}_{\boldsymbol{B}}\right) \Phi & =\left(\boldsymbol{A}_{\boldsymbol{\alpha}_{Q}} \times \boldsymbol{U}_{\boldsymbol{B}}\right)\left(1+P^{2}\right)^{-n-1}\left(1+P^{2}\right)^{n+1} \Phi \\
& =\left(\boldsymbol{A}_{\boldsymbol{\alpha}_{Q}} \times E(B) \boldsymbol{U}_{\mathbb{R}^{n}}\left(1+P^{2}\right)^{-n-1}\right)\left(1+P^{2}\right)^{n+1} \Phi .
\end{aligned}
$$

In the latter expression we can proceed to the limit $B \nearrow \mathbb{R}^{n}$ according to the second half of part (iv) of Lemma 2.1, since the projections $E(B)$ converge strongly to 1 in this limit. In view of relation (2.2), this proves the existence of the first type of integrals,

$$
\begin{aligned}
\int \alpha_{Q x}(A) d E(x) \Phi & \doteq \lim _{B \nearrow \mathbb{R}^{n}} \int_{B} \alpha_{Q x}(A) d E(x) \Phi \\
& =\left(\boldsymbol{A}_{\boldsymbol{\alpha}_{Q}} \times \boldsymbol{U}_{\mathbb{R}^{n}}\left(1+P^{2}\right)^{-n-1}\right)\left(1+P^{2}\right)^{n+1} \Phi
\end{aligned}
$$

Part (iii) and the first half of part (iv) of Lemma 2.1 imply that the functions $x \mapsto$ $\alpha_{x}\left(\boldsymbol{A}_{\boldsymbol{\alpha}_{Q}} \times \boldsymbol{U}_{\mathbb{R}^{n}}\left(1+P^{2}\right)^{-n-1}\right)=U(x) \boldsymbol{A}_{\boldsymbol{\alpha}_{Q}} U(x)^{-1} \times \boldsymbol{U}_{\mathbb{R}^{n}}\left(1+P^{2}\right)^{-n-1}$ are elements of $\mathcal{C}^{\infty}$, since $x, y \mapsto\left(U(x) \boldsymbol{A}_{\alpha_{Q}} U(x)^{-1}\right)(y)=\alpha_{x+Q y}(A)$ is smooth in both variables. So it is also clear that the domain $\mathcal{D}$ is stable under the action of the integrals.

For the proof of existence of the second type of integrals, we make use of the fact that the functions $x \mapsto \alpha_{x}\left(\left(1+P^{2}\right)^{n+1} A\left(1+P^{2}\right)^{-n-1}\right), A \in \mathcal{C}^{\infty}$ are elements of $\mathcal{C}^{\infty} .^{3}$ Thus, by the first half of part (iv) of Lemma 2.1, we get for $\Phi \in \mathcal{D}$,

$$
\begin{aligned}
\left(\boldsymbol{U}_{\boldsymbol{B}} \times \boldsymbol{A}_{\boldsymbol{\alpha}_{Q}}\right) \Phi= & \left(\boldsymbol{U}_{\boldsymbol{B}} \times \boldsymbol{A}_{\boldsymbol{\alpha}_{Q}}\right)\left(1+P^{2}\right)^{-n-1}\left(1+P^{2}\right)^{n+1} \Phi \\
= & \left(\boldsymbol{U}_{\boldsymbol{B}} \times\left(1+P^{2}\right)^{-n-1}\left(1+P^{2}\right)^{n+1} \boldsymbol{A}_{\boldsymbol{\alpha}_{Q}}\left(1+P^{2}\right)^{-n-1}\right)\left(1+P^{2}\right)^{n+1} \Phi \\
= & \left(\boldsymbol{U}_{\mathbb{R}^{n}} E(B)\left(1+P^{2}\right)^{-n-1} \times\left(1+P^{2}\right)^{n+1} \boldsymbol{A}_{\boldsymbol{\alpha}_{Q}}\left(1+P^{2}\right)^{-n-1}\right) \\
& \times\left(1+P^{2}\right)^{n+1} \Phi,
\end{aligned}
$$

where in the latter expression we can proceed again to the strong limit $E(B) \nearrow 1$ if $B \nearrow \mathbb{R}^{n}$. In view of relation (2.2), this proves existence of the strong limits

$$
\begin{aligned}
\int d E(x) \alpha_{Q x}(A) \Phi \doteq & \lim _{B \nearrow \mathbb{R}^{n}} \int_{B} d E(x) \alpha_{Q x}(A) \Phi \\
= & \left(\boldsymbol{U}_{\mathbb{R}^{n}}\left(1+P^{2}\right)^{-n-1} \times\left(1+P^{2}\right)^{n+1} \boldsymbol{A}_{\boldsymbol{\alpha}_{Q}}\left(1+P^{2}\right)^{-n-1}\right) \\
& \times\left(1+P^{2}\right)^{n+1} \Phi .
\end{aligned}
$$

3 This can be seen by pulling the components of $P$ through from the left to the right of $A$, noticing that their commutators with $A$ yield derivatives of $A$. 
By a similar argument as before, one finds that the domain $\mathcal{D}$ is stable under the action of the second type of integrals, as well.

It follows at once from the preceding results and Lemma 2.1 that one can conveniently present the two types of integrals on the domain $\mathcal{D}$ in terms of the strong limits

$$
\begin{aligned}
& \int \alpha_{Q x}(A) d E(x) \Phi=\lim _{\varepsilon \rightarrow 0}(2 \pi)^{-n} \iint d x d y f(\varepsilon x, \varepsilon y) e^{-i x y} \alpha_{Q x}(A) U(y) \Phi, \\
& \int d E(x) \alpha_{Q x}(A) \Phi=\lim _{\varepsilon \rightarrow 0}(2 \pi)^{-n} \iint d x d y f(\varepsilon x, \varepsilon y) e^{-i x y} U(x) \alpha_{Q y}(A) \Phi .
\end{aligned}
$$

We shall make use of these relations throughout the subsequent analysis.

In the following, we limit ourselves to the particularly interesting case where the matrix $Q$ entering into the definition of the integrals is skew symmetric relative to the chosen bilinear form on $\mathbb{R}^{n}$. It is understood without further mention that the integrals are defined on the common stable domain $\mathcal{D}$.

Lemma 2.2. Let $Q$ be any real skew symmetric matrix on $\mathbb{R}^{n}$ and let $A \in \mathcal{C}^{\infty}$. Then

(i) $\int d E(x) \alpha_{Q x}(A)=\int \alpha_{Q x}(A) d E(x)$ and

(ii) $\left(\int \alpha_{Q x}(A) d E(x)\right)^{*} \supset \int \alpha_{Q x}\left(A^{*}\right) d E(x)$.

Proof. (i) As pointed out above, one has for $\Phi \in \mathcal{D}$,

$$
\int \alpha_{Q x}(A) d E(x) \Phi=\lim _{\varepsilon \rightarrow 0}(2 \pi)^{-n} \iint d x d y f(\varepsilon x, \varepsilon y) e^{-i x y} \alpha_{Q x}(A) U(y) \Phi .
$$

The integration can be restricted to the submanifold $(\operatorname{ker} Q)^{\perp} \times(\operatorname{ker} Q)^{\perp}$, since the remaining integrals merely produce factors of $2 \pi$. Substituting $x \rightarrow x+Q^{-1} y$ and taking into account that $Q^{-1}$ is skew symmetric, one gets

$$
\begin{aligned}
& \iint d x d y f(\varepsilon x, \varepsilon y) e^{-i x y} \alpha_{Q x}(A) U(y) \\
& =\iint d x d y g(\varepsilon x, \varepsilon y) e^{-i x y} U(y) \alpha_{Q x}(A) \\
& =\iint d x d y g(\varepsilon y, \varepsilon x) e^{-i x y} U(x) \alpha_{Q y}(A),
\end{aligned}
$$

where $g(x, y) \doteq f\left(x+Q^{-1} y, y\right)$. In the limit $\varepsilon \rightarrow 0$ one obtains

$$
\lim _{\varepsilon \rightarrow 0}(2 \pi)^{-n} \iint d x d y g(\varepsilon y, \varepsilon x) e^{-i x y} U(x) \alpha_{Q y}(A) \Phi=\int d E(x) \alpha_{Q x}(A) \Phi,
$$

proving assertion (i).

(ii) For the proof of the second assertion, note that

$$
\begin{aligned}
& \left(\iint d x d y f(\varepsilon x, \varepsilon y) e^{-i x y} \alpha_{Q x}(A) U(y)\right)^{*} \\
& =\iint d x d y \overline{f(\varepsilon x, \varepsilon y)} e^{i x y} U(-y) \alpha_{Q x}\left(A^{*}\right) \\
& =\iint d x d y \overline{f(\varepsilon y,-\varepsilon x)} e^{-i x y} U(x) \alpha_{Q y}\left(A^{*}\right) .
\end{aligned}
$$


Hence, for $\Psi, \Phi \in \mathcal{D}$,

$$
\begin{aligned}
\left\langle\Psi, \int \alpha_{Q x}(A) d E(x) \Phi\right\rangle & =\lim _{\varepsilon \rightarrow 0}(2 \pi)^{-n}\left\langle\Psi, \iint d x d y f(\varepsilon x, \varepsilon y) e^{-i x y} \alpha_{Q x}(A) U(y) \Phi\right\rangle \\
& =\lim _{\varepsilon \rightarrow 0}(2 \pi)^{-n}\left\langle\iint d x d y \overline{f(\varepsilon y,-\varepsilon x)} e^{-i x y} U(x) \alpha_{Q y}\left(A^{*}\right) \Psi, \Phi\right\rangle \\
& =\left\langle\int d E(x) \alpha_{Q x}\left(A^{*}\right) \Psi, \Phi\right\rangle .
\end{aligned}
$$

The assertion now follows from the preceding step.

We may therefore meaningfully declare the following definition as in [13].

Definition 2.3. Let $Q$ be a real skew symmetric matrix on $\mathbb{R}^{n}$ and let $A \in \mathcal{C}^{\infty}$. The corresponding warped convolution $A_{Q}$ of $A$ is defined on the domain $\mathcal{D}$ by means of the preceding results according to

$$
A_{Q} \doteq \int d E(x) \alpha_{Q x}(A)=\int \alpha_{Q x}(A) d E(x) .
$$

In particular, $1_{Q}=1$.

We shall next show that the warped convolution provides a representation of the algebra $\left(\mathcal{C}^{\infty}, \times_{Q}\right)$ defined by $A \mapsto \pi_{Q}(A) \doteq A_{Q}$. The argument proceeds through a number of steps. It is apparent from the definition that the map $\pi_{Q}$ is linear and, by the second part of the preceding lemma, we have $\pi_{Q}(A)^{*} \supset \pi_{Q}\left(A^{*}\right)$. The proof that $\pi_{Q}$ is also multiplicative requires more work.

Lemma 2.4. Let $Q$ be a real skew symmetric matrix on $\mathbb{R}^{n}$ and let $A, B \in \mathcal{C}^{\infty}$. Then (understood as an equality on $\mathcal{D}$ )

$$
A_{Q} B_{Q}=\left(A \times{ }_{Q} B\right)_{Q},
$$

where $\times_{Q}$ denotes the Rieffel product on $\mathcal{C}^{\infty}$. In other words,

$$
\pi_{Q}(A) \pi_{Q}(B)=\pi_{Q}\left(A \times_{Q} B\right) .
$$

Proof. Let $f, g$ be test functions as in Lemma 2.1. Recalling that $A \times Q B \in \mathcal{C}^{\infty}$ can be approximated in norm according to

$$
A \times{ }_{Q} B=\lim _{\delta \rightarrow 0}(2 \pi)^{-n} \iint d v d w f(\delta v, \delta w) e^{-i v w} \alpha_{Q v}(A) \alpha_{w}(B),
$$

one finds for $\Phi \in \mathcal{D}$,

$$
\begin{aligned}
(2 \pi)^{2 n}\left(A \times{ }_{Q} B\right)_{Q} \Phi= & \lim _{\varepsilon, \delta \rightarrow 0} \iiint \int d v d w d x d y f(\delta v, \delta w) g(\varepsilon x, \varepsilon y) \\
& \times e^{-i v w-i x y} \alpha_{Q v+Q x}(A) \alpha_{w+Q x}(B) U(y) \Phi,
\end{aligned}
$$

in the sense of strong convergence. Similarly, one has

$$
\begin{aligned}
(2 \pi)^{2 n} A_{Q} B_{Q} \Phi= & \lim _{\varepsilon, \delta \rightarrow 0} \iiint \int_{\int} d v d w d x d y g(\varepsilon v, \varepsilon w) f(\delta x, \delta y) \\
& \times e^{-i v w-i x y} \alpha_{Q v}(A) U(w) \alpha_{Q x}(B) U(y) \Phi .
\end{aligned}
$$


In both cases the limits are to be performed in the given order. In order to see that these limits coincide, one rewrites the two integrals. For the first one, by substituting $(v, w) \rightarrow(v-x, w-Q x)$ and bearing in mind that $Q$ is skew symmetric, one obtains

$$
\begin{aligned}
& \iiint \int d v d w d x d y f(\delta v, \delta w) g(\varepsilon x, \varepsilon y) e^{-i v w-i x y} \alpha_{Q v+Q x}(A) \alpha_{w+Q x}(B) U(y) \Phi \\
& =\iiint \int d v d w d x d y h_{\delta, \varepsilon}(v, w, x, y) e^{-i v w-i x(y+Q v-w)} \alpha_{Q v}(A) \alpha_{w}(B) U(y) \Phi,
\end{aligned}
$$

where $h_{\delta, \varepsilon}(v, w, x, y) \doteq f(\delta(v-x), \delta(w-Q x)) g(\varepsilon x, \varepsilon y)$. For the second integral, by substituting $(w, y) \rightarrow(w-Q x, y+Q x-w)$ and making use again of the fact that $Q$ is skew symmetric, one finds

$$
\begin{aligned}
& \iiint \int d v d w d x d y g(\varepsilon v, \varepsilon w) f(\delta x, \delta y) e^{-i v w-i x y} \alpha_{Q v}(A) U(w) \alpha_{Q x}(B) U(y) \Phi \\
& =\iiint \int d v d w d x d y k_{\delta, \varepsilon}(v, w, x, y) e^{-i v w-i x(y+Q v-w)} \alpha_{Q v}(A) \alpha_{w}(B) U(y) \Phi,
\end{aligned}
$$

where $k_{\delta, \varepsilon}(v, w, x, y)=f(\delta x, \delta(y+Q x-w)) g(\varepsilon v, \varepsilon(w-Q x))$. Thus the two integrals coincide apart from the mollifying test functions $h_{\delta, \varepsilon}$ and $k_{\delta, \varepsilon}$, respectively.

In order to show that the two integrals have the same limits, one proceeds as in the proof of part (i) of Lemma 2.1. Again one finds by Fourier transformation that for any given polynomial $L$ on $\mathbb{R}^{4 n}$ there is a corresponding polynomial $P$ such that

$$
L(v, w, x, y) e^{-i v w-i x(y+Q v-w)}=P\left(-\partial_{v},-\partial_{w},-\partial_{x},-\partial_{y}\right) e^{-i v w-i x(y+Q v-w)} .
$$

A convenient choice for $L$ is given by

$$
L(v, w, x, y)=\left(L_{n}(v) L_{n}(w) L_{n}(x) L_{n}(y)\right)^{-1},
$$

where $L_{n}$ are the mollifiers introduced before. With this choice one gets by partial integration, setting $\boldsymbol{u} \doteq(v, w, x, y), d \boldsymbol{u}=d v d w d x d y$ and $\boldsymbol{\partial} \doteq\left(\partial_{v}, \partial_{w}, \partial_{x}, \partial_{y}\right)$,

$$
\begin{aligned}
& \iiint \int d \boldsymbol{u} h_{\delta, \varepsilon}(\boldsymbol{u}) e^{-i v w-i x(y+Q v-w)} \alpha_{Q v}(A) \alpha_{w}(B) U(y) \Phi \\
& =\iiint \int d \boldsymbol{u} e^{-i v w-i x(y+Q v-w)} P(\boldsymbol{\partial}) h_{\delta, \varepsilon}(\boldsymbol{u}) L(\boldsymbol{u})^{-1} \alpha_{Q v}(A) \alpha_{w}(B) U(y) \Phi .
\end{aligned}
$$

The derivatives in the second line are well-defined, since $A, B \in \mathcal{C}^{\infty}$ and $\Phi \in \mathcal{D}$. Moreover, all derivatives of $\boldsymbol{u} \mapsto L(\boldsymbol{u})^{-1}$ are absolutely integrable, and the derivatives of $\boldsymbol{u} \mapsto h_{\delta, \varepsilon}(\boldsymbol{u})$ produce factors of $\delta$ and $\varepsilon$, respectively. Thus in the limit of small $\delta$ and $\varepsilon$ one can replace in the above integral the test function $h_{\delta, \varepsilon}$ by its value at the origin, i.e. 1. The same argument applies if one replaces $h_{\delta, \varepsilon}$ by $k_{\delta, \varepsilon}$, proving equality of the limits of the respective integrals.

At this point, the operators $\pi_{Q}(A)=A_{Q}, A \in \mathcal{C}^{\infty}$, are well-defined only on the dense, invariant domain $\mathcal{D}$, defining there a ${ }^{*}$-algebra. We next show that they may be extended to bounded operators on $\mathcal{H}$, in contradiction to an assertion made in [13]. In the proof we make use of the fact that the algebra $\left(\mathcal{C}^{\infty}, \times_{Q}\right)$ admits a $C^{*}$-norm $\|\cdot\| Q$, cf. [28, Ch. 4]. It thus can be completed to a $C^{*}$-algebra, denoted by $\left(\mathcal{C}_{Q}, \times{ }_{Q}\right)$, to which the group of automorphisms $\alpha_{x}, x \in \mathbb{R}^{n}$, extends in a strongly continuous manner [28, Prop. 5.11].

As is well known, every positive element of a $C^{*}$-algebra has a positive square root in the algebra. We need here the following more detailed information. 
Lemma 2.5. Let $A \in \mathcal{C}^{\infty}$ be strictly positive in $\left(\mathcal{C}_{Q}, \times_{Q}\right)$, i.e. $A-\delta 1=B^{*} \times{ }_{Q} B$ for some $\delta>0$ and $B \in\left(\mathcal{C}_{Q}, \times_{Q}\right)$. Then its positive square root $\sqrt{A} \in\left(\mathcal{C}_{Q}, \times_{Q}\right)$ is also an element of $\mathcal{C}^{\infty}$.

Proof. The form of $A$ implies that its spectrum is contained in the interval $\left[\delta,\|A\|_{Q}\right]$. As the square root $z \mapsto \sqrt{z}$ is holomorphic in a complex neighborhood of this region and $\mathcal{C}^{\infty}$ is closed under the holomorphic calculus [28, Cor. 7.6], it follows that $\sqrt{A} \in \mathcal{C}^{\infty}$.

With the help of this lemma we can show now that the operators $\pi_{Q}(A)=A_{Q}$, $A \in \mathcal{C}^{\infty}$ are bounded. For the operators $(\delta+a)^{2} 1-A^{*} \times{ }_{Q} A, a \doteq\|A\|_{Q}$, are elements of $\mathcal{C}^{\infty}$ and strictly positive in $\left(\mathcal{C}_{Q}, \times_{Q}\right)$ for every $\delta>0$. Thus their positive square roots $B \doteq \sqrt{(\delta+a)^{2} 1-A^{*} \times{ }_{Q} A} \in\left(\mathcal{C}_{Q}, \times_{Q}\right)$ are elements of $\mathcal{C}^{\infty}$ according to the preceding lemma. Bearing in mind the properties of $\pi_{Q}$ established thus far, we therefore have for any $\Phi \in \mathcal{D}$,

$$
\begin{aligned}
& (\delta+a)^{2}\|\Phi\|^{2}-\left\|\pi_{Q}(A) \Phi\right\|^{2} \\
& =\left\langle\Phi, \pi_{Q}\left((\delta+a)^{2} 1-A^{*} \times_{Q} A\right) \Phi\right\rangle=\left\langle\Phi, \pi_{Q}\left(B^{*} \times_{Q} B\right) \Phi\right\rangle=\left\|\pi_{Q}(B) \Phi\right\|^{2} \geq 0
\end{aligned}
$$

where we made use of the fact that $B^{*}=B$ since $B$ is positive. Hence we obtain $\left\|\pi_{Q}(A) \Phi\right\| \leq\left(\|A\|_{Q}+\delta\right)\|\Phi\|, \Phi \in \mathcal{D}$. Since $\mathcal{D}$ is dense in $\mathcal{H}$ and $\delta>0$ was arbitrary, we conclude that the operators $\pi_{Q}(A)$ can be extended to the whole Hilbert space with operator norms satisfying the bound

$$
\left\|\pi_{Q}(A)\right\| \leq\|A\|_{Q}, \quad A \in \mathcal{C}^{\infty} .
$$

Moreover, it follows from this estimate and the preceding results that the representation $\pi_{Q}: \mathcal{C}^{\infty} \rightarrow \mathcal{B}(\mathcal{H})$ can be continuously extended to a representation of the $C^{*}$-algebra $\left(\mathcal{C}_{Q}, \times_{Q}\right)$ on $\mathcal{H}$. We summarize these findings.

Theorem 2.6. The map

$$
\pi_{Q}(A) \doteq A_{Q}, \quad A \in \mathcal{C}^{\infty}
$$

extends to a representation of the Rieffel-deformed $C^{*}$-algebra $\left(\mathcal{C}_{Q}, \times_{Q}\right)$ on $\mathcal{H}$. In particular, one has the bound

$$
\left\|\pi_{Q}(A)\right\| \leq\|A\|_{Q}, \quad A \in\left(\mathcal{C}_{Q}, \times_{Q}\right) .
$$

Example. Of particular interest in physics are the cases where the spectrum of $U$ contains an atomic part. Without loss of generality one may then assume that $\{0\}$ is part of the atomic spectrum with corresponding invariant vector $\Omega .{ }^{4}$ Since the algebra $\mathcal{C}^{\infty}$ is weakly dense in $\mathcal{B}(\mathcal{H})$, it is clear that $\Omega$ is cyclic for $\mathcal{C}^{\infty}$; moreover, because of the invariance of $\Omega$ under the action of $U$, one also has $\mathcal{C}^{\infty} \Omega \subset \mathcal{D}$. Within this setting the

\footnotetext{
4 Note that proceeding from the group $U(x)$ to the group $U_{q}(x)=e^{i q x} U(x), x \in \mathbb{R}^{n}$, merely amounts to a translation $A \rightarrow \alpha_{Q q}(A)$ of the operators $A$ in the original warped convolution.
} 
relation between the warped convolutions and the Rieffel deformations can be exhibited quite easily. For, as a consequence of the invariance of $\Omega$, one obtains for $A, B \in \mathcal{C}^{\infty}$,

$$
\begin{aligned}
A_{Q} B \Omega & =\lim _{\varepsilon \rightarrow 0}(2 \pi)^{-n} \iint d x d y e^{-i x y} f(\varepsilon x, \varepsilon y) \alpha_{Q x}(A) U(y) B \Omega \\
& =\lim _{\varepsilon \rightarrow 0}(2 \pi)^{-n} \iint d x d y e^{-i x y} f(\varepsilon x, \varepsilon y) \alpha_{Q x}(A) \alpha_{y}(B) \Omega \\
& =\left(A \times{ }_{Q} B\right) \Omega .
\end{aligned}
$$

In particular,

$$
A_{Q} \Omega=A \Omega, \quad A \in \mathcal{C}^{\infty} .
$$

Making use of the associativity of the product $\times{ }_{Q}$ on $\mathcal{C}^{\infty}$, it is therefore clear that for $A, B, C \in \mathcal{C}^{\infty}$,

$$
A_{Q} B_{Q} C \Omega=A_{Q}\left(B \times{ }_{Q} C\right) \Omega=\left(A \times{ }_{Q} B \times{ }_{Q} C\right) \Omega=\left(A \times{ }_{Q} B\right)_{Q} C \Omega .
$$

We return now to the discussion of the general case and exhibit further interesting properties of the representations $\pi_{Q}$ introduced above.

Proposition 2.7. Let $\pi_{Q}$ be the representation of the $C^{*}$-algebra $\left(\mathcal{C}_{Q}, \times_{Q}\right)$ established by the preceding theorem.

(i) $\pi_{Q}$ is $\alpha$-covariant, i.e. for any $A \in\left(\mathcal{C}_{Q}, \times_{Q}\right)$

$$
\pi_{Q}\left(\alpha_{x}(A)\right)=U(x) \pi_{Q}(A) U(x)^{-1}, \quad x \in \mathbb{R}^{n} .
$$

(ii) $\pi_{Q}$ induces a bijective map of $\mathcal{C}^{\infty}$ onto itself.

(iii) $\pi_{Q}$ is faithful, i.e. $\left\|\pi_{Q}(A)\right\|=\|A\|_{Q}, A \in\left(\mathcal{C}_{Q}, \times_{Q}\right)$.

(iv) $\pi_{Q}$ is irreducible.

Proof. (i) Let $A \in \mathcal{C}^{\infty}$. Since the domain $\mathcal{D}$ is stable under the action of the unitaries $U(x)$, relation (2.3) and Lemma 2.1 imply $U(x) A_{Q} U(x)^{-1}=\left(\alpha_{x}(A)\right)_{Q}$, proving the assertion for $A \in \mathcal{C}^{\infty}$. The continuity properties of $\pi_{Q}$ and the automorphic action of $\alpha_{x}$ on $\left(\mathcal{C}_{Q}, \times_{Q}\right)$ then yield assertion (i).

(ii) According to [28, Thm. 7.1], the smooth elements of $\left(\mathcal{C}_{Q}, \times_{Q}\right)$ are exactly the elements of $\mathcal{C}^{\infty}$. It therefore follows from the continuity of the map $\pi_{Q}$ that the functions $x \mapsto \pi_{Q}\left(\alpha_{x}(A)\right), A \in \mathcal{C}^{\infty}$, are smooth; hence $\pi_{Q}(A)=A_{Q} \in \mathcal{C}^{\infty}$ for $A \in \mathcal{C}^{\infty}$. The proof that $\pi_{Q} \uparrow \mathcal{C}^{\infty}$ is bijective requires a computation: In view of the preceding observation, one may apply the warping procedure with underlying matrix $-Q$ to the operator $A_{Q}$, giving $\left(A_{Q}\right)_{-Q}$. Now according to relation (2.4) one has on the domain $\mathcal{D}$,

$$
\begin{aligned}
& (2 \pi)^{2 n}\left(A_{Q}\right)_{-Q} \\
& =\lim _{\varepsilon, \delta \rightarrow 0} \iiint \int d v d w d x d y f(\varepsilon v, \varepsilon w) f(\delta x, \delta y) e^{-i v w-i x y} \alpha_{Q x-Q v}(A) U(y) U(w),
\end{aligned}
$$

where the limits are to be performed in the given order. Substituting $(v, w) \rightarrow(x-v$, $w-y$ ), the integral can be transformed into

$$
\iiint \int d v d w d x d y f(\varepsilon(x-v), \varepsilon(w-y)) f(\delta x, \delta y) e^{i v w-i x w-i y v} \alpha_{Q v}(A) U(w) .
$$


As the $x, y$-integration in the latter integral involves only ordinary functions, it is straightforward to compute its limit for $\delta \rightarrow 0$, giving

$$
(2 \pi)^{n} \iint d v d w(1 / \varepsilon)^{2 n} \widehat{f}(w / \varepsilon,-v / \varepsilon) e^{-i v w} \alpha_{Q v}(A) U(w),
$$

where $\widehat{f}$ denotes the Fourier transform of $f$. It is also apparent that the latter expression converges to $(2 \pi)^{2 n} A$ as $\varepsilon \rightarrow 0$. Hence $\left(A_{Q}\right)_{-Q}=A$ for $A \in \mathcal{C}^{\infty}$. Now if $\pi_{Q}(A)=A_{Q}=0$, it follows that $A=\left(A_{Q}\right)_{-Q}=0$, so $\pi_{Q}\left\lceil\mathcal{C}^{\infty}\right.$ is injective; similarly, interchanging the role of $Q$ and $-Q$, one has $\pi_{Q}\left(A_{-Q}\right)=\left(A_{-Q}\right)_{Q}=A$, so $\pi_{Q}\left\lceil\mathcal{C}^{\infty}\right.$ is also surjective.

(iii) Since $\pi_{Q}$ is $\alpha$-covariant, its kernel $\operatorname{ker} \pi_{Q}$ is $\alpha$-invariant. Hence, in view of the strongly continuous action of $\alpha$ on $\left(\mathcal{C}^{\infty}, \times_{Q}\right)$, the space $\operatorname{ker} \pi_{Q} \cap \mathcal{C}^{\infty}$ is dense in ker $\pi_{Q}$. But this space coincides with $\{0\}$, since $\pi_{Q}\left\lceil\mathcal{C}^{\infty}\right.$ is injective according to the preceding result. Consequently, $\left\|\pi_{Q}(\cdot)\right\|$ defines a $C^{*}$-norm on $\left(\mathcal{C}_{Q}, \times_{Q}\right)$, which must coincide with $\|\cdot\|_{Q}$ because of the uniqueness of such norms.

(iv) The final assertion follows from the fact that $\pi_{Q}\left\lceil\mathcal{C}^{\infty}\right.$ is surjective. So its range contains $\mathcal{C}^{\infty}$, which is weakly dense in $\mathcal{B}(\mathcal{H})$.

Let us turn now to the case of an abstractly given $C^{*}$-dynamical system $\left(\mathcal{A}, \mathbb{R}^{n}\right)$ equipped with some strongly continuous representation $\alpha: \mathbb{R}^{n} \rightarrow$ Aut $\mathcal{A}$. Denoting by $\mathcal{A}^{\infty}$ the smooth elements of $\mathcal{A}$, one obtains by arguments given by Rieffel [28] and sketched at the end of Sect. 2.1 a deformed ${ }^{*}$-algebra $\left(\mathcal{A}^{\infty}, \times^{Q}\right)$ with $C^{*}$-norm $\|\cdot\|^{Q}$ for any given skew symmetric matrix $Q$. Its $C^{*}$-completion will be $\operatorname{denoted}\left(\mathcal{A}^{Q},{ }^{Q}\right)$. Here we have used $Q$ as an upper index in order to distinguish the abstract setting from the concrete one used thus far.

Let $(\pi, \mathcal{H})$ be an $\alpha$-covariant representation of $\mathcal{A}$ on a Hilbert space $\mathcal{H}$, i.e. on $\mathcal{H}$ there exists a weakly continuous unitary representation $U$ of $\mathbb{R}^{n}$ such that

$$
U(x) \pi(A) U(x)^{-1}=\pi\left(\alpha_{x}(A)\right), \quad A \in \mathcal{A} .
$$

Consequently $\pi\left(\mathcal{A}^{\infty}\right) \subset \mathcal{C}^{\infty}$, so one can define for any $A, B \in \mathcal{A}^{\infty}$ the operators $\pi(A){ }_{Q} \in \mathcal{C}^{\infty}$ and the product $\pi(A) \times{ }_{Q} \pi(B)$; moreover, $\pi(A) \times{ }_{Q} \pi(B)=\pi\left(A \times{ }^{Q} B\right)$.

After having established the properties of the warping procedure on $\mathcal{C}^{\infty}$, it is almost evident that the covariant representation $(\pi, \mathcal{H})$ of $\mathcal{A}$ induces a covariant representation $\left(\pi^{Q}, \mathcal{H}\right)$ of $\left(\mathcal{A}^{Q}, \times^{Q}\right)$. It is fixed by setting

$$
\pi^{Q}(A) \doteq \pi(A)_{Q}, \quad A \in \mathcal{A}^{\infty}
$$

By Theorem 2.6, the operators $\pi^{Q}(A)$ are bounded. Moreover, it follows from Lemma 2.2 that

$$
\pi^{Q}(A)^{*}=\left(\pi(A)_{Q}\right)^{*}=\left(\pi(A)^{*}\right)_{Q}=\pi\left(A^{*}\right)_{Q}=\pi^{Q}\left(A^{*}\right) .
$$

Similarly, Lemma 2.4 implies

$\pi^{Q}(A) \pi^{Q}(B)=\pi(A){ }_{Q} \pi(B)_{Q}=(\pi(A) \times Q \pi(B))_{Q}=\pi\left(A \times{ }^{Q} B\right)_{Q}=\pi^{Q}\left(A \times{ }^{Q} B\right)$.

Finally, one may employ the analogue of Lemma 2.5 in the abstract setting and the reasoning thereafter to obtain $\left\|\pi^{Q}(A)\right\| \leq\|A\|^{Q}, A \in \mathcal{A}^{\infty}$. Hence the homomorphism 
$\pi^{Q}: \mathcal{A}^{\infty} \rightarrow \mathcal{B}(\mathcal{H})$ can be extended by continuity to a representation of $\left(\mathcal{A}^{Q}, \times^{Q}\right)$, as claimed. From the first part of Proposition 2.7 it follows that

$$
\begin{aligned}
& U(x) \pi^{Q}(A) U(x)^{-1} \\
& \quad=U(x) \pi(A)_{Q} U(x)^{-1}=\left(U(x) \pi(A) U(x)^{-1}\right)_{Q}=\pi\left(\alpha_{x}(A)\right)_{Q}=\pi^{Q_{(}}\left(\alpha_{x}(A)\right),
\end{aligned}
$$

for all $A \in \mathcal{A}^{\infty}$. So the representation $\pi^{Q}$ is also covariant, hence $\pi^{Q}\left(\mathcal{A}^{\infty}\right) \subset \mathcal{C}^{\infty}$. Depending on the properties of the chosen representation $(\pi, \mathcal{H})$ of $\mathcal{A}$, the map $\pi Q$ : $\mathcal{A}^{\infty} \rightarrow \mathcal{C}^{\infty}$ may not be injective or surjective. But according to part (ii) of the preceding proposition one has $\pi(A)_{Q}=0$ if and only if $\pi(A)=0, A \in \mathcal{A}^{\infty}$. Furthermore, in view of the continuity of the action $\alpha$ on $\mathcal{A}$ and $\mathcal{A}^{Q}$, the inclusions $\operatorname{ker} \pi \cap \mathcal{A}^{\infty} \subset \operatorname{ker} \pi$ and ker $\pi^{Q} \cap \mathcal{A}^{\infty} \subset \operatorname{ker} \pi^{Q}$ are dense in the norms $\|\cdot\|$ and $\|\cdot\| Q$, respectively. Thus it follows that $\pi^{Q}$ is faithful if and only if $\pi$ is faithful.

Theorem 2.8. Let $(\pi, \mathcal{H})$ be an $\alpha$-covariant representation of the $C^{*}$-algebra $\mathcal{A}$. The homomorphism $\pi^{Q}: \mathcal{A}^{\infty} \rightarrow \mathcal{B}(\mathcal{H})$, fixed by the relation

$$
\pi^{Q}(A) \doteq \pi(A) Q, \quad A \in \mathcal{A}^{\infty},
$$

extends continuously to an $\alpha$-covariant representation of the $C^{*}-\operatorname{algebra}\left(\mathcal{A}^{Q}, \times^{Q}\right)$. Moreover, $\pi^{Q}$ is faithful if and only if $\pi$ is faithful.

So the warping method provides a representation of the deformed algebras in the same Hilbert space as the undeformed algebra, enabling the direct comparison of deformed operators corresponding to different $Q$. This point will prove to be useful in the physical context treated below.

2.3. Further properties of warped convolutions. Even though the warped convolutions may be viewed as merely generating certain specific representations of Rieffel algebras, it will be advantageous to base the subsequent discussion directly on them without referring to the Rieffel setting. The reasons for this are threefold: (a) It will be necessary to deal with subalgebras of the algebra of smooth operators which are not invariant under the automorphic action of the translations. So there is no corresponding Rieffel algebra, but the warping procedure is still meaningful. (b) It will be necessary to consider warped operators $A_{Q}, A_{Q^{\prime}}^{\prime}$ and their sums and products for different matrices $Q, Q^{\prime}$. Such operations can be carried out in the framework of warped convolutions more easily than in the Rieffel setting, where one has to use Hilbert modules instead of Hilbert spaces. (c) We shall need to establish algebraic properties of the warped operators arising from spectral properties of the unitary representation $U$, which are not available in the Rieffel setting.

Returning to the Hilbert space framework, we first exhibit some general covariance properties of the warped convolutions, $c f$. [13]. To this end we consider (anti)unitary operators $V$ whose adjoint actions on the translations $U$ induce linear transformations of $\mathbb{R}^{n}$. It follows at once that for any such $V$ the algebra $\mathcal{C}^{\infty}$ is stable under the corresponding adjoint action, $V \mathcal{C}^{\infty} V^{-1}=\mathcal{C}^{\infty}$, and $V \mathcal{D}=\mathcal{D}$. The following result is the first instance where we must deal with warped convolutions for different choices of the underlying matrix $Q$. 
Proposition 2.9. Let $V$ be a unitary or antiunitary operator on $\mathcal{H}$ such that $V U(x) V^{-1}=$ $U(M x), x \in \mathbb{R}^{n}$, for some invertible matrix $M$. Then, for $A \in \mathcal{C}^{\infty}$,

$$
V A_{Q} V^{-1}=\left(V A V^{-1}\right)_{\sigma M Q M^{T}},
$$

where $M^{T}$ is the transpose of $M$ with respect to the chosen bilinear form, $\sigma=1$ if $V$ is unitary and $\sigma=-1$ if $V$ is antiunitary.

Proof. Making use of relation (2.4) for real $f$, one commences from the equalities of strong integrals

$$
\begin{aligned}
& V \iint d x d y e^{-i x y} f(\varepsilon x, \varepsilon y) \alpha_{Q x}(A) U(y) V^{-1} \\
& =\iint d x d y e^{-i \sigma x y} f(\varepsilon x, \varepsilon y) \alpha_{M Q x}\left(V A V^{-1}\right) U(M y) \\
& =\iint d x d y e^{-i x y} f\left(\varepsilon \sigma M^{T} x, \varepsilon M^{-1} y\right) \alpha_{\sigma M Q M^{T} x}\left(V A V^{-1}\right) U(y),
\end{aligned}
$$

where the last equality is obtained by substituting $(x, y) \rightarrow\left(\sigma M^{T} x, M^{-1} y\right)$. Applying these relations to any vector $\Phi \in \mathcal{D}$ and taking into account $V^{-1} \mathcal{D}=\mathcal{D}$, the assertion follows in the limit of small $\varepsilon$.

Next, we establish a result which is fundamental for the applications to physics. We shall show that the warped convolutions preserve certain specific commutation properties of the operators in $\mathcal{C}^{\infty}$ for appropriate choices of the underlying skew symmetric matrices depending on the spectrum of the representation $U$ [13].

Proposition 2.10. Let $A, B \in \mathcal{C}^{\infty}$ be operators such that $\left[\alpha_{Q x}(A), \alpha_{-Q y}(B)\right]=0$ for all $x, y \in \operatorname{sp} U$. Then

$$
\left[A_{Q}, B_{-Q}\right]=0 .
$$

Proof. Returning to the definition of the warped convolutions by the spectral calculus and making use of Lemma 2.2, one finds for vectors $\Phi, \Psi$ with compact spectral support

$$
\left\langle\Phi, A_{Q} B_{-Q} \Psi\right\rangle=\lim _{F, F^{\prime} \nearrow 1}\left\langle\Phi,\left(\int d E(x) F \alpha_{Q x}(A)\right)\left(\int \alpha_{-Q y}(B) F^{\prime} d E(y)\right) \Psi\right\rangle,
$$

where $F, F^{\prime}$ are finite-dimensional projections. Now

$$
\begin{aligned}
\langle\Phi & \left.,\left(\int d E(x) F \alpha_{Q x}(A)\right)\left(\int \alpha_{-Q y}(B) F^{\prime} d E(y)\right) \Psi\right\rangle \\
= & \iint\left\langle\Phi, d E(x) F \alpha_{Q x}(A) \alpha_{-Q y}(B) F^{\prime} d E(y) \Psi\right\rangle \\
& =\iint\left\langle\Phi, d E(x) F \alpha_{-Q y}(B) \alpha_{Q x}(A) F^{\prime} d E(y) \Psi\right\rangle,
\end{aligned}
$$

where the step from the first to the second line is justified by the fact that the given expression can be decomposed into a finite sum of product measures multiplied with smooth functions. The second step is a consequence of the commutativity properties of $A$ and $B$. Introducing the notation $\boldsymbol{u}=(v, w, x, y) \in \mathbb{R}^{4 n}$ and picking any test function 
$\boldsymbol{u} \mapsto h(\boldsymbol{u})$ which is equal to 1 at 0 , it follows from the spectral representation of $U$ that the latter integral is equal to

$$
\lim _{\varepsilon \rightarrow 0}(2 \pi)^{-2 n} \iiint \int d \boldsymbol{u} h(\varepsilon \boldsymbol{u}) e^{-i v x-i y w}\left\langle\Phi, U(v) F \alpha_{-Q y}(B) \alpha_{Q x}(A) F^{\prime} U(w) \Psi\right\rangle .
$$

Adopting now the arguments and notation in the final part of the proof of Lemma 2.4, one finds that for the polynomial $L(2.5)$ there exists a corresponding polynomial $P$ such that

$$
\begin{aligned}
& \iiint \int d \boldsymbol{u} h(\varepsilon \boldsymbol{u}) e^{-i v x-i y w}\left\langle\Phi, U(v) F \alpha_{-Q y}(B) \alpha_{Q x}(A) F^{\prime} U(w) \Psi\right\rangle \\
& =\iiint \int d \boldsymbol{u} e^{-i v x-i y w} P(\boldsymbol{\partial}) h(\varepsilon \boldsymbol{u}) L(\boldsymbol{u})^{-1}\left\langle\Phi, U(v) F \alpha_{-Q y}(B) \alpha_{Q x}(A) F^{\prime} U(w) \Psi\right\rangle .
\end{aligned}
$$

After having performed the differentiations in the last integral, one sees by an application of the dominated convergence theorem that the composite limit $\varepsilon \rightarrow 0, F, F^{\prime} \nearrow 1$ is independent of the order in which the individual limits are carried out and also does not depend on the choice of $h$. Thus one has, in particular,

$$
\begin{aligned}
& \left\langle\Phi, A_{Q} B_{-Q} \Psi\right\rangle \\
& =\lim _{\varepsilon \rightarrow 0}(2 \pi)^{-2 n} \iiint \int d \boldsymbol{u} h(\varepsilon \boldsymbol{u}) e^{-i v x-i y w}\left\langle\Phi, U(v) \alpha_{-Q y}(B) \alpha_{Q x}(A) U(w) \Psi\right\rangle .
\end{aligned}
$$

As before, one takes advantage of the fact that the integration may be restricted to the submanifold $(\operatorname{ker} Q)^{\perp} \times \cdots \times(\operatorname{ker} Q)^{\perp} \subset \mathbb{R}^{4 n}$, since the remaining integrals merely produce factors of $2 \pi$. So the preceding integral can be recast as

$$
\begin{aligned}
& \iiint \int d \boldsymbol{u} e^{-i v x-i y w} h(\varepsilon \boldsymbol{u})\left\langle\Phi, U(v) \alpha_{-Q y}(B) \alpha_{Q x}(A) U(w) \Psi\right\rangle \\
& =\iiint \int d \boldsymbol{u} e^{-i v x-i y w} h(\varepsilon \boldsymbol{u})\left\langle\Phi, U(w) \alpha_{-Q y+v-w}(B) \alpha_{Q x+v-w}(A) U(v) \Psi\right\rangle \\
& =\iiint \int d \boldsymbol{u} e^{-i v x-i y w} k(\varepsilon \boldsymbol{u})\left\langle\Phi, U(w) \alpha_{-Q y}(B) \alpha_{Q x}(A) U(v) \Psi\right\rangle,
\end{aligned}
$$

where $k(v, w, x, y)=h\left(v, w, x-Q^{-1}(v-w), y+Q^{-1}(v-w)\right)$. The last equality is the result of the substitution $(x, y) \rightarrow\left(x-Q^{-1}(v-w), y+Q^{-1}(v-w)\right.$, under which $e^{-i v x-i y w}$ does not change because of the skew symmetry of $Q$. Proceeding to the limit of small $\varepsilon$, one obtains by relation (2.4) and Lemma 2.2,

$$
\begin{aligned}
& \lim _{\varepsilon \rightarrow 0}(2 \pi)^{-2 n} \iiint \int d \boldsymbol{u} e^{-i v x-i y w} k(\varepsilon \boldsymbol{u})\left\langle\Phi, U(w) \alpha_{-Q y}(B) \alpha_{Q x}(A) U(v) \Psi\right\rangle \\
& \quad=\left\langle\Phi, B_{-} A_{Q} \Psi\right\rangle .
\end{aligned}
$$

This shows that $\left\langle\Phi, A_{Q} B_{-Q} \Psi\right\rangle=\left\langle\Phi, B_{-Q} A_{Q} \Psi\right\rangle$. Since $\Phi, \Psi$ were arbitrary elements of a dense set of vectors, the assertion now follows.

We finally discuss the structure of the family of maps given by the warped convolutions. According to Proposition 2.7 (ii), these maps act bijectively on $\mathcal{C}^{\infty}$ and therefore can be composed and have inverses. In fact, they form a group which is homomorphic to $\mathbb{R}^{n(n-1) / 2}$, as can be seen from the next proposition. 
Proposition 2.11. Let $Q_{1}, Q_{2}$ be skew symmetric matrices. Then

$$
\left(A_{Q_{1}}\right)_{Q_{2}}=A_{Q_{1}+Q_{2}}, \quad A \in \mathcal{C}^{\infty} .
$$

Proof. To begin, note that for any continuous bounded function $f$ of the generator $P$ of $U$ one has $A_{Q} f(P)=(A f(P))_{Q}$, as a consequence of relation (2.3) and part (iv) of Lemma 2.1. Let $\Phi \in \mathcal{D}$ be any vector with compact spectral support and let $f$ be a test function such that $f(P) \Phi=\Phi$. It follows that $\left(A_{Q_{1}}\right)_{Q_{2}} \Phi=\left(A_{Q_{1}} f(P)\right)_{Q_{2}} f(P) \Phi$. Picking nets of finite-dimensional projections $F, F^{\prime}$ converging to 1 , making use of the spectral calculus, which implies $f(P) d E(z)=f(z) d E(z), z \in \mathbb{R}^{n}$, and recalling the definition of the warped convolutions, one obtains in the sense of weak convergence

$$
\left(A_{Q_{1}}\right)_{Q_{2}} \Phi=\lim _{F^{\prime} \nearrow 1, F \nearrow 1} \iint f(x) f(y) \alpha_{Q_{1} x+Q_{2} y}(A) F d E(x) F^{\prime} d E(y) \Phi .
$$

Here the limits are taken in the given order and the (strong) limit $F \nearrow 1$ has been interchanged with the $y$-integration by an application of the dominated convergence theorem. Since the function $x, y \mapsto f(x) f(y) \alpha_{Q_{1} x+Q_{2} y}(A)$ is smooth and rapidly decreasing in norm, one can interchange the limits. The product measure $d E(x) F^{\prime} d E(y)$ converges weakly in the sense of distributions to $\delta(x-y) d x d E(y)$ as $F^{\prime} \nearrow 1$, where $\delta(x-y) d x$ is the Dirac measure at $y$; hence one obtains

$$
\left(A_{Q_{1}}\right)_{Q_{2}} \Phi=\lim _{F \nearrow 1} \int \alpha_{\left(Q_{1}+Q_{2}\right) x}(A) F d E(x) f(P)^{2} \Phi=A_{Q_{1}+Q_{2}} \Phi .
$$

The desired conclusion then follows, because the space of vectors $\Phi$ with compact spectral support is dense in $\mathcal{H}$.

Note that this result does not entail a composition law of the representations $\pi_{Q}$ of the Rieffel algebras, since their ranges do not, in general, fit with their respective domains.

Further Results. Most of the preceding results can be established in a setting of unbounded operators. One proceeds again from a continuous unitary representation $U$ of $\mathbb{R}^{n}$ and considers the ${ }^{*}$-algebra $\mathcal{F}$ of all operators $F$ for which there is some $n_{F} \in \mathbb{N}$ such that the functions $x \mapsto\left(1+P^{2}\right)^{-n_{F}} \alpha_{x}(F)\left(1+P^{2}\right)^{-n_{F}}$ are arbitrarily often differentiable in norm. The operators $F \in \mathcal{F}$ are defined on the domain $\mathcal{D}$ and leave it invariant. Making use of the fact that there is a version of Lemma 2.1 in this setting, one can define the Rieffel product $\times_{Q}$ on $\mathcal{F}$; the warped convolutions of the elements of $\mathcal{F}$ can be defined as well and are elements of $\mathcal{F}$. Moreover, Lemmas 2.2 and 2.4 hold without changes, so the warped convolutions define an (unbounded) ${ }^{*}$-representation of $\left(\mathcal{F}, \times_{Q}\right)$, and Propositions 2.9, 2.10 and 2.11 hold as well. We refrain from giving the proofs here.

\section{Warped Convolutions and Borchers Triples}

We consider now warped convolutions in the context of Borchers triples, invented by Borchers [3] for the construction and analysis of relativistic quantum field theories. This setting is, on the one hand, more restrictive than the preceding one, since one deals with unitary representations $U$ of the translations $\mathbb{R}^{n}, n \geq 2$, with certain specific spectral properties. On the other hand, one considers subalgebras of $\mathcal{B}(\mathcal{H})$ on which the adjoint action $\alpha$ of $U$ merely induces endomorphisms for semigroups of translations in the set $\mathcal{W} \doteq\left\{x=\left(x_{0}, x_{1}, \ldots, x_{n-1}\right) \in \mathbb{R}^{n}: x_{1} \geq\left|x_{0}\right|\right\}$. 
Definition 3.1. A Borchers triple $(\mathcal{R}, U, \Omega$ ) (relative to $\mathcal{W}$ ) consists of

(a) a von Neumann algebra $\mathcal{R} \subset \mathcal{B}(\mathcal{H})$,

(b) a weakly continuous unitary representation $U$ of $\mathbb{R}^{n}$ on $\mathcal{H}$ whose spectrum is contained in the closed forward light cone $V_{+}=\left\{p=\left(p_{0}, p_{1}, \ldots, p_{n-1}\right) \in \mathbb{R}^{n}\right.$ : $p_{0} \geq \sqrt{p_{1}^{2}+\cdots+p_{n-1}^{2}}$ and which satisfies $\alpha_{x}(\mathcal{R}) \subset \mathcal{R}, x \in \mathcal{W}$,

(c) and a unit vector $\Omega \in \mathcal{H}$ which is invariant under the action of $U$ and is cyclic and separating for $\mathcal{R}$.

By condition (c), Tomita-Takesaki theory [30,31] is applicable to the pair $(\mathcal{R}, \Omega)$, and we shall denote by $\Delta, J$ the associated modular operator and involution. In this context Borchers [3] proved the following remarkable theorem (see [15] for a simpler proof).

Theorem 3.2. Let $(\mathcal{R}, U, \Omega)$ be a Borchers triple relative to $\mathcal{W}$. Denoting by $\vartheta(t)$, $t \in \mathbb{R}$, and $j$ the transformations acting on $x=\left(x_{0}, x_{1}, \ldots x_{n-1}\right) \in \mathbb{R}^{n}$ by

$$
\begin{aligned}
\vartheta(t) x & \doteq\left(\cosh (2 \pi t) x_{0}+\sinh (2 \pi t) x_{1}, \sinh (2 \pi t) x_{0}+\cosh (2 \pi t) x_{1}, x_{2}, \ldots, x_{n-1}\right), \\
j x & \doteq\left(-x_{0},-x_{1}, x_{2}, \ldots x_{n-1}\right),
\end{aligned}
$$

one has

(i) $\Delta^{i t} U(x) \Delta^{-i t}=U(\vartheta(t) x)$ for $x \in \mathbb{R}^{n}$ and $t \in \mathbb{R}$,

(ii) $J U(x) J=U(j x)$ for $x \in \mathbb{R}^{n}$.

Moreover, $\left(\mathcal{R}^{\prime}, U, \Omega\right)$ is a Borchers triple relative to $-\mathcal{W}$, where $\mathcal{R}^{\prime}=J \mathcal{R} J$ is the commutant of $\mathcal{R}$.

Proof. The assertion for $n=2$ is proven in [3]. Setting $x_{\perp}=\left(0,0, x_{2}, \ldots, x_{n-1}\right)$, conditions (b), (c) in Definition 3.1 imply $U\left(x_{\perp}\right) \mathcal{R} U\left(x_{\perp}\right)^{-1}=\mathcal{R}$ and $U\left(x_{\perp}\right) \Omega=\Omega$. The uniqueness of the modular objects then entails that $\Delta$ and $J$ both commute with all $U\left(x_{\perp}\right)$, completing the proof in the general case.

We shall show now that the family of Borchers triples is stable under the deformations induced by warped convolutions corresponding to certain specific choices of the skew symmetric matrix ${ }^{5} Q$. Moreover, the modular objects of the deformed triples coincide with those of the original one. This observation is of relevance in quantum field theory, which will be discussed at the end of this section.

We begin with some technical remarks. Let $\mathcal{C}^{\infty}$ be, as above, the ${ }^{*}$-algebra of all smooth elements in $\mathcal{B}(\mathcal{H})$ under the adjoint action of the translations and let $\mathcal{R}^{\infty}=$ $\mathcal{R} \cap \mathcal{C}^{\infty}$. In view of condition (b) in Definition 3.1, one obtains elements of $\mathcal{R}^{\infty}$ by smoothing any element $R \in \mathcal{R}$ with Schwartz test functions $f$ having support in $\mathcal{W}$,

$$
R(f) \doteq \int d x f(x) \alpha_{x}(R) .
$$

These weak integrals are elements of $\mathcal{R}^{\infty}$ since, by construction, they are smooth and contained in the von Neumann algebra $\mathcal{R}$. Choosing sequences $f_{n}$ of test functions with support in $\mathcal{W}$ which approximate the Dirac measure at 0 , one sees that $\mathcal{R}^{\infty}$ is dense in $\mathcal{R}$ in the strong operator topology, and consequently $\Omega$ is cyclic for $\mathcal{R}^{\infty}$. By the same reasoning one finds that $\Omega$ is also cyclic for $\mathcal{R}^{\prime \infty} \doteq \mathcal{R}^{\prime} \cap \mathcal{C}^{\infty}$.

5 Having in mind applications to quantum field theory, we choose henceforth the Lorentz product $x y=$ $x_{0} y_{0}-\sum_{m=1}^{n-1} x_{m} y_{m}, x, y \in \mathbb{R}^{n}$, as the bilinear form on $\mathbb{R}^{n}$. 
Now let $Q$ be any real skew symmetric matrix on $\mathbb{R}^{n}$ which is $\mathcal{W}$-compatible in the sense that $Q V_{+} \subset \mathcal{W}$. This constraint on $Q$ will become important in the following. The corresponding warped operators $A_{Q}, A \in \mathcal{R}^{\infty}$, are defined as in the previous section. Since they are bounded and satisfy $A_{Q}{ }^{*}=A^{*}{ }_{Q}$, they generate a von Neumann algebra, called a warped algebra for short. With a slight abuse of notation, we write

$$
\mathcal{R}_{Q} \doteq\left\{A_{Q}: A \in \mathcal{R}^{\infty}\right\}^{\prime \prime}
$$

For the proof that the warped triple $\left(\mathcal{R}_{Q}, U, \Omega\right)$ is again a Borchers triple, we note that, as a consequence of Proposition 2.9, one has $\alpha_{x}\left(\mathcal{R}_{Q}\right)=\alpha_{x}(\mathcal{R})_{Q} \subset \mathcal{R}_{Q}$ for $x \in \mathcal{W}$. So condition (b) in Definition 3.1 is satisfied. Furthermore, since $\Omega$ is cyclic for $\mathcal{R}^{\infty}$, it is also cyclic for $\mathcal{R}_{Q}$ as a consequence of Eq. (2.7). In order to see that $\Omega$ is separating for $\mathcal{R}_{Q}$, let $A \in \mathcal{R}^{\infty}, A^{\prime} \in \mathcal{R}^{\prime \infty}$. Then $\left[\alpha_{x}(A), \alpha_{y}\left(A^{\prime}\right)\right]=0$ for $x \in \mathcal{W}, y \in-\mathcal{W}$, and taking into account that $Q \operatorname{sp} U \subset Q V_{+} \subset \mathcal{W}$, it follows from Proposition 2.10 that $\left[A_{Q}, A^{\prime}{ }_{-Q}\right]=0$. Thus $\left(\mathcal{R}^{\prime}\right)_{-Q} \subset\left(\mathcal{R}_{Q}\right)^{\prime}$. But Eq. (2.7) implies that $\Omega$ is cyclic for $\left(\mathcal{R}^{\prime}\right)_{-Q}$ and thus a fortiori for $\left(\mathcal{R}_{Q}\right)^{\prime}$. Hence $\Omega$ is separating for $\mathcal{R}_{Q}$, and condition (c) in Definition 3.1 holds as well.

Theorem 3.3. Let $(\mathcal{R}, U, \Omega)$ be a Borchers triple relative to $\mathcal{W}$ and let $Q$ be $\mathcal{W}$-compatible. Then the resulting warped triple $\left(\mathcal{R}_{Q}, U, \Omega\right)$ is also a Borchers triple relative to $\mathcal{W}$.

In view of this theorem, we may apply modular theory to the warped triple. We shall show next that the corresponding modular objects coincide with the original ones. To this end we need the following technical lemma.

Lemma 3.4. Let $(\mathcal{R}, U, \Omega)$ be a Borchers triple relative to $\mathcal{W}$ and let $S=J \Delta^{1 / 2}$ be the corresponding Tomita conjugation given by the closure of the map

$$
S A \Omega=A^{*} \Omega, \quad A \in \mathcal{R} .
$$

Then the subdomain $\mathcal{R}^{\infty} \Omega$ is a core for $S$.

Proof. Let $R \in \mathcal{R}$ and let $f_{n}$ be a sequence of real test functions with support in $\mathcal{W}$ such that in the sense of strong convergence $\lim _{n} R\left(f_{n}\right) \Omega=R \Omega$, cf. relation (3.1) and the remarks thereafter. Since $R\left(f_{n}\right) \in \mathcal{R}^{\infty} \subset \mathcal{R}$ and

$$
\lim _{n} S R\left(f_{n}\right) \Omega=\lim _{n} R\left(f_{n}\right)^{*} \Omega=\lim _{n} R^{*}\left(f_{n}\right) \Omega=R^{*} \Omega=S R \Omega,
$$

the conclusion follows, because $\mathcal{R} \Omega$ is a core for $S$ by definition.

We are now in a position to establish the invariance of the modular objects of Borchers triples under the warping procedure.

Theorem 3.5. Let $(\mathcal{R}, U, \Omega)$ be a Borchers triple relative to $\mathcal{W}$ with modular objects $\Delta, J$, and let $Q$ be a $\mathcal{W}$-compatible matrix. Then the modular objects $\Delta_{Q}, J_{Q}$ associated with the warped triple $\left(\mathcal{R}_{Q}, U, \Omega\right)$ coincide with those of the original triple, i.e.

$$
\Delta_{Q}=\Delta, \quad J_{Q}=J
$$


Proof. Let $S_{Q}$ be the Tomita conjugation associated with the warped triple $\left(\mathcal{R}_{Q}, U, \Omega\right)$ and let $S$ be the Tomita conjugation associated with $(\mathcal{R}, U, \Omega)$. Since $A_{Q} \in \mathcal{R}_{Q}$ for $A \in \mathcal{R}^{\infty}$, Eq. (2.7) and Lemma 2.2 imply

$$
S_{Q} A \Omega=S_{Q} A_{Q} \Omega=\left(A_{Q}\right)^{*} \Omega=\left(A^{*}\right)_{Q} \Omega=A^{*} \Omega=S A \Omega .
$$

According to the preceding lemma, $\mathcal{R}^{\infty} \Omega$ is a core for $S$, hence $S_{Q} \supset S$. By the Tomita-Takesaki theory [30,31], the adjoint $S_{Q}{ }^{*}$ of $S_{Q}$ is theS Tomita conjugation associated with $\left(\left(\mathcal{R}_{Q}\right)^{\prime}, U, \Omega\right)$, and similarly $S^{*}$ is the Tomita conjugation associated with $\left(\mathcal{R}^{\prime}, U, \Omega\right)$. It was shown in the proof of Theorem 3.3 that $\left(\mathcal{R}^{\prime}\right)_{-Q} \subset\left(\mathcal{R}_{Q}\right)^{\prime}$. Thus, as $A^{\prime}{ }_{-Q} \in \mathcal{R}^{\prime}{ }_{-Q}$ for $A^{\prime} \in \mathcal{R}^{\prime \infty}$, one obtains by another application of Eq. (2.7) and Lemma 2.2,

$$
S_{Q}{ }^{\prime} A^{\prime} \Omega=S_{Q}{ }^{*} A_{-Q}^{\prime} \Omega=\left(A^{\prime}{ }_{-Q}\right)^{*} \Omega=\left(A^{\prime *}\right)_{-Q} \Omega=A^{\prime *} \Omega=S^{*} A^{\prime} \Omega .
$$

By the preceding lemma $\mathcal{R}^{\prime \infty} \Omega$ is a core for $S^{*}$, hence $S_{Q}{ }^{*} \supset S^{*}$ and consequently $S \supset S_{Q}$, since both conjugations are closed operators. Thus $S_{Q}=S$ and, by the uniqueness of the polar decomposition, the desired conclusion follows.

An immediate consequence of this theorem is the observation that

$$
\mathcal{R}_{Q}^{\prime}=\mathcal{R}_{-Q}^{\prime}
$$

Indeed, Theorem 3.2 and Proposition 2.9 imply $J \mathcal{R}_{Q} J=(J \mathcal{R} J)_{-j Q j}$ and it is also straightforward to verify that $j Q j=Q$ for any $\mathcal{W}$-admissible matrix $Q$. Since $J=J_{Q}$, the asserted equation then follows from Tomita-Takesaki theory.

Let us discuss now the physical significance of these findings. As was pointed out in [3], Theorem 3.2 allows one to use the Borchers triple $(\mathcal{R}, U, \Omega)$ as a building block for the construction of a quantum field theory in two spacetime dimensions. Identifying the cone $\mathcal{W} \subset \mathbb{R}^{2}$ defined above with the corresponding wedge shaped region in twodimensional Minkowski space, one interprets $\mathcal{A}(\mathcal{W}) \doteq \mathcal{R}$ as the algebra generated by observables which are localized in $\mathcal{W}$. Moreover, noticing that the transformations $\vartheta(t)$, $t \in \mathbb{R}$, and $j$ introduced in Theorem 3.2 have the geometrical meaning of Lorentz boosts and spacetime reflection, respectively, one can consistently extend the representation $U$ of the translations $\mathbb{R}^{2}$ to a continuous (anti)unitary representation of the proper Poincaré group $\mathcal{P}_{+}$. It is given by

$$
U(\lambda) \doteq U(x) J^{\sigma} \Delta^{i t}, \quad \lambda=\left(x, j^{\sigma} \vartheta(t)\right) \in \mathcal{P}_{+},
$$

where $x \in \mathbb{R}^{2}, t \in \mathbb{R}$ and $\sigma \in\{0,1\}$. Thus $J$ represents the PCT-operator. With the help of this representation one can define the algebras generated by observables in the transformed wedge regions $\lambda \mathcal{W}, \lambda \in \mathcal{P}_{+}$by setting

$$
\mathcal{A}(\lambda \mathcal{W}) \doteq U(\lambda) \mathcal{R} U(\lambda)^{-1}, \quad \lambda \in \mathcal{P}_{+} .
$$

This definition is consistent, since the stability group of the wedge $\mathcal{W}$ in $\mathcal{P}_{+}$consists of the boosts $\vartheta(t), t \in \mathbb{R}$, whose corresponding automorphic action leaves the algebra $\mathcal{R}$ invariant according to Tomita-Takesaki theory. The resulting assignment $\mathcal{W} . \mapsto \mathcal{A}(\mathcal{W}$.) of wedge regions to algebras defines a net (pre-cosheaf) on $\mathbb{R}^{2}$. It is Poincaré covariant by construction and causal. In fact, since $j$ maps the wedge $\mathcal{W}$ onto its spacelike complement $\mathcal{W}^{\prime}=-\mathcal{W}$, one has

$$
\mathcal{A}\left(\mathcal{W}^{\prime}\right)=U(j) \mathcal{A}(\mathcal{W}) U(j)^{-1}=J \mathcal{R} J=\mathcal{R}^{\prime}=\mathcal{A}(\mathcal{W})^{\prime},
$$


where the third equality follows from Tomita-Takesaki theory. So the observables in spacelike separated wedges commute, in accordance with the principle of Einstein causality. In this way any Borchers triple defines a relativistic quantum field theory in two spacetime dimensions, $c f$. [3] for more details.

The upshot of these considerations is the insight that, as a consequence of the preceding three theorems, the warped triples $\left(\mathcal{R}_{Q}, U, \Omega\right)$ generate in the same manner another causal and covariant net $\mathcal{W} . \mapsto \mathcal{A}_{Q}(\mathcal{W}$.) by setting

$$
\mathcal{A}_{Q}(\lambda \mathcal{W}) \doteq U(\lambda) \mathcal{R}_{Q} U(\lambda)^{-1}, \quad \lambda \in \mathcal{P}_{+}
$$

Thus the warping procedure provides a tool for the consistent deformation of twodimensional quantum field theories without changing the underlying representation of the Poincare group. We shall further elaborate on this observation in the next section.

\section{Warped Convolutions in Quantum Field Theory}

In this section we examine applications of the warping procedure to relativistic quantum field theories in more than two spacetime dimensions. Thus we interpret $\mathbb{R}^{n}, n>2$, as Minkowski space equipped with the standard metric fixed by the Lorentz product, $c f$. footnote 5 . The identity component of its isometry group, the Poincare group, is the semidirect product $\mathcal{P}_{+}^{\uparrow}=\mathbb{R}^{n} \rtimes \mathcal{L}_{+}^{\uparrow}$ of the spacetime translations $\mathbb{R}^{n}$ and the proper orthochronous Lorentz transformations $\mathcal{L}_{+}^{\uparrow}$.

In a manner similar to the preceding section, we describe the theories in the algebraic setting of local quantum physics [20] by a qualified version of the concept of Borchers triple. Additional constraints arise since, on the one hand, the group generated by the translations, along with the boosts and reflection emerging from the modular structure of the triple, does not act transitively on the set of wedge regions in $\mathbb{R}^{n}$ if $n>2$. The smallest subgroup of the Poincaré group which fulfills this condition is $\mathcal{P}_{+}^{\uparrow}$. So one needs from the outset an action of this group on the underlying algebra $\mathcal{R}$, which one interprets again as the algebra of observables localized in the given wedge region $\mathcal{W} \doteq\left\{x=\left(x_{0}, x_{1}, \ldots, x_{n-1}\right) \in \mathbb{R}^{n}: x_{1} \geq\left|x_{0}\right|\right\}$. On the other hand, one must ensure that this action is consistent with the principle of Einstein causality, according to which observables in spacelike separated regions must commute. The resulting consistency conditions can be expressed in terms of the triple in an evident manner, $c f$. [2, Prop. 7.3.22]. They lead to the concept of a causal Borchers triple.

Definition 4.1. A causal Borchers triple $(\mathcal{R}, U, \Omega)$ relative to $\mathcal{W}$ consists of

(a) a von Neumann algebra $\mathcal{R} \subset \mathcal{B}(\mathcal{H})$,

(b) a weakly continuous unitary representation $U$ of $\mathcal{P}_{+}^{\uparrow}$ such that, $\lambda \in \mathcal{P}_{+}^{\uparrow}$,

$$
\begin{aligned}
& U(\lambda) \mathcal{R} U(\lambda)^{-1} \subset \mathcal{R} \text { if } \lambda \mathcal{W} \subset \mathcal{W}, \\
& U(\lambda) \mathcal{R} U(\lambda)^{-1} \subset \mathcal{R}^{\prime} \text { if } \lambda \mathcal{W} \subset \mathcal{W}^{\prime},
\end{aligned}
$$

and the spectrum of the abelian subgroup $U \uparrow \mathbb{R}^{n}$ of the spacetime translations is contained in the closed forward lightcone $V_{+}$,

(c) and a unit vector $\Omega \in \mathcal{H}$, describing the vacuum, which is invariant under the action of $U$ and is cyclic and separating for $\mathcal{R}$. 
Remark. In two spacetime dimensions any Borchers triple determines a causal Borchers triple by the modular construction in the preceding section. As there is no element in $\mathcal{P}_{+}^{\uparrow}$ which maps the wedge $\mathcal{W}$ into its spacelike (causal) complement $\mathcal{W}^{\prime}$, the second constraint in condition (b) is trivially satisfied in this case. In order to flip the wedge one needs the spacetime reflection $j$, which is an element of $\mathcal{P}_{+} \supset \mathcal{P}_{+}^{\uparrow}$. As we have seen, its corresponding action on $\mathcal{R}$ is consistent with Einstein causality as a consequence of modular theory. In higher dimensions one either has to posit causality from the outset, as we do, or one has to impose additional constraints on the modular structure of the triple which imply it, $c f$. $[4,5,7,10,19]$.

With the above input one can define the algebras corresponding to arbitrary regions in $\mathbb{R}^{n}$ in a straightforward manner, which we briefly recall. Making use of the fact that $\mathcal{P}_{+}^{\uparrow}$ acts transitively on the wedge regions, one begins with the wedge algebras by setting

$$
\mathcal{A}(\lambda \mathcal{W}) \doteq U(\lambda) \mathcal{R} U(\lambda)^{-1}, \quad \lambda \in \mathcal{P}_{+}^{\uparrow}
$$

This definition is consistent, since $\lambda_{1} \mathcal{W}=\lambda_{2} \mathcal{W}$ implies that the transformation $\lambda_{2}^{-1} \lambda_{1}$ is an element of the stability group of $\mathcal{W}$, and $\mathcal{R}$ is stable under the adjoint action of the corresponding unitary operators according to the first part of condition (b). Similarly, if $\lambda_{1} \mathcal{W} \subset \lambda_{2} \mathcal{W}$, it follows that $U\left(\lambda_{2}^{-1} \lambda_{1}\right) \mathcal{R} U\left(\lambda_{2}^{-1} \lambda_{1}\right)^{-1} \subset \mathcal{R}$, hence $\mathcal{A}\left(\lambda_{1} \mathcal{W}\right) \subset$ $\mathcal{A}\left(\lambda_{2} \mathcal{W}\right)$. Thus the family of wedge algebras complies with the condition of isotony. The wedge algebras also transform covariantly under the adjoint action of the representation $U$ by their very definition. Moreover, if $\lambda_{1} \mathcal{W} \subset\left(\lambda_{2} \mathcal{W}\right)^{\prime}$, then $U\left(\lambda_{2}^{-1} \lambda_{1}\right) \mathcal{R} U\left(\lambda_{2}^{-1} \lambda_{1}\right)^{-1}$ $\subset \mathcal{R}^{\prime}$ according to the second part of condition (b). Hence $\mathcal{A}\left(\lambda_{1} \mathcal{W}\right) \subset \mathcal{A}\left(\lambda_{2} \mathcal{W}\right)^{\prime}$ in accordance with Einstein causality. The algebras corresponding to arbitrary causally closed convex regions $\mathcal{O} \subset \mathbb{R}^{n}$ are determined from the wedge algebras $\mathcal{A}(\mathcal{W}$.) by setting $\mathcal{A}(\mathcal{O})=\bigcap_{\mathcal{W}} \supset \mathcal{O} \mathcal{A}(\mathcal{W}$. $)$. It is apparent that the resulting assignment $\mathcal{O} \mapsto \mathcal{A}(\mathcal{O})$ inherits the structure of a causal and covariant net on $\mathbb{R}^{n}$, i.e. of a local quantum theory [20]. It should be noted, however, that within the present general framework the algebras corresponding to bounded regions may happen to be trivial. We shall comment on the physical significance of this possibility at the end of this section.

We now want to use our warping procedure to deform causal Borchers triples. Additional constraints on the underlying skew symmetric matrices arise due to the extra conditions imposed on such triples. In fact, $Q$ must have the following form with respect to the coordinates chosen in the specification of the wedge $\mathcal{W} \subset \mathbb{R}^{n}$ :

$$
Q \doteq\left(\begin{array}{ccccc}
0 & \zeta & 0 & \cdots & 0 \\
\zeta & 0 & 0 & \cdots & 0 \\
0 & 0 & 0 & \cdots & 0 \\
\vdots & \vdots & \vdots & \ddots & \vdots \\
0 & 0 & 0 & \cdots & 0
\end{array}\right)
$$

for fixed $\zeta \geq 0$. In the special but physically most interesting case of $n=4$ dimensions, one can admit matrices of the more general form

$$
Q \doteq\left(\begin{array}{cccc}
0 & \zeta & 0 & 0 \\
\zeta & 0 & 0 & 0 \\
0 & 0 & 0 & \eta \\
0 & 0 & -\eta & 0
\end{array}\right)
$$


for fixed $\zeta \geq 0, \eta \in \mathbb{R}$. Note that these matrices are skew symmetric with respect to the Lorentz product. The following facts pointed out in [17] are crucial for the consistent deformation of the triples and, in turn, determine the choice of the admissible matrices $Q$ [17, Lemma 2].

(i) $Q V_{+} \subset \mathcal{W}$.

(ii) Let $\lambda=(x, \Lambda) \in \mathcal{P}_{+}^{\uparrow}$ be such that $\lambda \mathcal{W} \subset \mathcal{W}$. Then $\Lambda Q \Lambda^{T}=Q$.

(iii) Let $\lambda=(x, \Lambda) \in \mathcal{P}_{+}^{\uparrow}$ be such that $\lambda \mathcal{W} \subset \mathcal{W}^{\prime}$. Then $\Lambda Q \Lambda^{T}=-Q$.

Any matrix $Q$ with these properties is said to be $\mathcal{W}$-admissible (qualifying the notion of $\mathcal{W}$-compatibility introduced in the preceding section).

Given a causal Borchers triple $(\mathcal{R}, U, \Omega)$ relative to $\mathcal{W}$, we proceed as in the preceding section and define for fixed $\mathcal{W}$-admissible matrix $Q$ the warped von Neumann algebra

$$
\mathcal{R}_{Q} \doteq\left\{A_{Q}: A \in \mathcal{R}^{\infty}\right\}^{\prime \prime}
$$

The corresponding warped triple $\left(\mathcal{R}_{Q}, U, \Omega\right)$ is again a causal Borchers triple. For the proof of this fact we make use of Proposition 2.9, according to which

$$
U(\lambda) A_{Q} U(\lambda)^{-1}=\left(U(\lambda) A U(\lambda)^{-1}\right)_{\Lambda Q \Lambda^{T},}, \quad \lambda=(x, \Lambda) \in \mathcal{P}_{+}^{\uparrow},
$$

for all $A \in \mathcal{C}^{\infty}$. Taking into account properties (ii) and (iii) of $Q$ given above, we conclude that

$$
\begin{aligned}
& U(\lambda) \mathcal{R}_{Q} U(\lambda)^{-1}=\left(U(\lambda) \mathcal{R} U(\lambda)^{-1}\right)_{Q} \subset \mathcal{R}_{Q} \text { if } \lambda \mathcal{W} \subset \mathcal{W}, \\
& U(\lambda) \mathcal{R}_{Q} U(\lambda)^{-1}=\left(U(\lambda) \mathcal{R} U(\lambda)^{-1}\right)_{-Q} \subset\left(\mathcal{R}^{\prime}\right)_{-Q} \text { if } \lambda \mathcal{W} \subset \mathcal{W}^{\prime} .
\end{aligned}
$$

But from Eq. (3.2) one has $\left(\mathcal{R}^{\prime}\right)_{-Q}=\left(\mathcal{R}_{Q}\right)^{\prime}$; hence the warped triple satisfies condition (b) in Definition 4.1. In the proof of Theorem 3.3, it was shown that $\Omega$ is cyclic and separating for $\mathcal{R}_{Q}$, so the triple also complies with condition (c).

Theorem 4.2. Let $(\mathcal{R}, U, \Omega)$ be a causal Borchers triple relative to $\mathcal{W}$ and let $Q$ be a $\mathcal{W}$-admissible matrix. The corresponding warped triple $\left(\mathcal{R}_{Q}, U, \Omega\right)$ is again a causal Borchers triple relative to $\mathcal{W}$.

So the deformations induced by the warped convolutions are consistent with the basic principles of local quantum physics. It is noteworthy that also certain more specific features persist under these deformations, such as the physically significant property of wedge duality. This property can be encoded into a maximality condition on the Borchers triple, which implies that the underlying algebra cannot be enlarged without coming into conflict with causality.

Definition 4.3. Let $(\mathcal{R}, U, \Omega)$ be a causal Borchers triple relative to $\mathcal{W}$. The triple is said to be maximally causal if $U(\lambda) \mathcal{R} U(\lambda)^{-1}=\mathcal{R}^{\prime}$ for any $\lambda \in \mathcal{P}_{+}^{\uparrow}$ such that $\lambda \mathcal{W}=\mathcal{W}^{\prime}$.

It immediately follows from the definition of the wedge algebras that, under these circumstances, $\mathcal{A}\left(\mathcal{W} .^{\prime}\right)=\mathcal{A}(\mathcal{W} \text {. })^{\prime}$ for all wedges $\mathcal{W}$., i.e. wedge duality obtains.

Proposition 4.4. Let $(\mathcal{R}, U, \Omega)$ be a maximally causal Borchers triple relative to $\mathcal{W}$ and let $Q$ be a $\mathcal{W}$-admissible matrix. Then the corresponding warped Borchers triple $\left(\mathcal{R}_{Q}, U, \Omega\right)$ is also maximally causal. 
Proof. Let $\lambda \in \mathcal{P}_{+}^{\uparrow}$ with $\lambda \mathcal{W}=\mathcal{W}^{\prime}$. Then property (iii) of the $\mathcal{W}$-admissible matrix $Q$, Proposition 2.9 and the maximality condition imply that

$$
U(\lambda) \mathcal{R}_{Q} U(\lambda)^{-1}=\left(U(\lambda) \mathcal{R} U(\lambda)^{-1}\right)_{-Q}=\mathcal{R}_{-Q}^{\prime}
$$

Equation (3.2) completes the proof.

Let us turn now to the question whether the deformed Borchers triples generate new theories. It is apparent that equivalent triples, as defined below, give rise to isomorphic nets of observable algebras and therefore must be identified.

Definition 4.5. Let $\left(\mathcal{R}_{1}, U_{1}, \Omega_{1}\right)$ and $\left(\mathcal{R}_{2}, U_{2}, \Omega_{2}\right)$ be two causal Borchers triples. The triples are equivalent if there exists an isometry $V: \mathcal{H}_{1} \rightarrow \mathcal{H}_{2}$ between the underlying Hilbert spaces such that $V \mathcal{R}_{1}=\mathcal{R}_{2} V, V U_{1}(\lambda)=U_{2}(\lambda) V$ for all $\lambda \in \mathcal{P}_{+}^{\uparrow}$, and $V \Omega_{1}=\Omega_{2}$.

Note that the algebras encountered in Borchers triples are generically isomorphic to the unique hyperfinite factor of type $\mathrm{III}_{1}$ and hence to each other. Thus the nontrivial requirement in the definition is the condition that the isometry $V$ intertwines, besides the algebras, the respective representations of the Poincaré group.

Although one may expect that the warped Borchers triples are generally inequivalent to the original ones, there does not yet exist an argument to that effect. It has been shown in $[13,17]$ that in theories describing massive particles the elastic scattering matrix changes under these deformations, thereby providing a rather indirect proof that the respective Borchers triples must be inequivalent. We present here an alternative argument, covering a large family of theories in more than two spacetime dimensions. It is based on the following lemma, whose proof is given in the Appendix. There we also comment on the additional physically meaningful spectral constraint on the translations made in the hypothesis.

Lemma 4.6. Let $(\mathcal{R}, U, \Omega)$ be a causal Borchers triple relative to $\mathcal{W} \subset \mathbb{R}^{n}, n \geq 3$, such that sp $U \uparrow \mathbb{R}^{n}$ contains some point in the interior of $V_{+}$and let $Q \neq 0$ be a $\mathcal{W}$-admissible matrix of the generic form (4.2). Then $\Omega$ is cyclic for at most one of the algebras $\bigcap_{\lambda \in \mathcal{N}} \alpha_{\lambda}(\mathcal{R})$ and $\bigcap_{\lambda \in \mathcal{N}} \alpha_{\lambda}\left(\mathcal{R}_{Q}\right)$, where $\mathcal{N}$ is any given neighborhood of the identity in $\mathcal{P}_{+}^{\uparrow}$.

The following observation about the relation between Borchers triples and their warped descendants is an immediate consequence of this result.

Proposition 4.7. Let $(\mathcal{R}, U, \Omega)$ be a causal Borchers triple relative to $\mathcal{W} \subset \mathbb{R}^{n}, n \geq 3$, such that sp $U \uparrow \mathbb{R}^{n}$ contains some point in the interior of $V_{+}$and let $\Omega$ be cyclic for $\bigcap_{\lambda \in \mathcal{N}} \alpha_{\lambda}(\mathcal{R})$ for some neighborhood $\mathcal{N}$ of the identity in $\mathcal{P}_{+}^{\uparrow}$. Then $\left(\mathcal{R}_{Q}, U, \Omega\right)$ and $(\mathcal{R}, U, \Omega)$ are inequivalent for any $\mathcal{W}$-admissible matrix $Q \neq 0$ of the generic form (4.2).

Proof. Let $V$ be some unitary operator which intertwines the two triples. Then $V \bigcap_{\lambda \in \mathcal{N}}$ $\alpha_{\lambda}(\mathcal{R}) V^{-1}=\bigcap_{\lambda \in \mathcal{N}} \alpha_{\lambda}\left(V \mathcal{R} V^{-1}\right)=\bigcap_{\lambda \in \mathcal{N}} \alpha_{\lambda}\left(\mathcal{R}_{Q}\right)$. Hence $\Omega=V \Omega$ is cyclic for the latter algebra as well, in conflict with the preceding lemma.

In the familiar examples of quantum field theories which have been rigorously constructed so far, such as (generalized) free field theories in physical spacetime and interacting field theories in lower dimensions [16], the vacuum $\Omega$ is known to be cyclic for 
the algebras affiliated with compact spacetime regions (Reeh-Schlieder property). Thus, applying the warping procedure to the corresponding Borchers triples, one ends up with inequivalent, i.e. new theories. However, the local algebras in the deformed theories no longer have the Reeh-Schlieder property, according to the preceding lemma. In fact, even for the algebras affiliated with pointed spacelike cones, which are of relevance in gauge theory [8], $\Omega$ is not cyclic. Thus in more than two spacetime dimensions the warped algebras can, in general, not be interpreted in terms of some underlying point fields.

Yet, as was pointed out in [13], the warped theories admit a meaningful physical interpretation with respect to noncommutative Minkowski space (Moyal space). In fact, the first examples of such theories appeared in that setting [17]. We recall that noncommutative Minkowski space is described by coordinate operators $X_{\mu}$ satisfying the commutation relations $\left[X_{\mu}, X_{v}\right]=i \theta_{\mu \nu} 1$, where $\theta_{\mu \nu}=-\theta_{\nu \mu}$ are real constants, $\mu, v=0,1, \ldots, n-1$. It is straightforward to verify that in more than two dimensions there always exist certain lightlike coordinates $X_{ \pm}$which commute and thus can be simultaneously diagonalized. Hence it should be possible to localize fields and observables with respect to these coordinates, thereby dislocalizing them in the remaining ones. In particular, the wedges $\mathcal{W}$ considered here are possible localization regions in noncommutative Minkowski space, whereas bounded regions and pointed spacelike cones are not. On the basis of this interpretation, the algebras corresponding to the latter regions are expected to be trivial, in line with the preceding lemma. Now, apart from the wedges, there are other cylindrical regions (such as the intersections of opposite wedges) which are possible localization regions. It is therefore an intriguing question whether the corresponding algebras in the warped theories are nontrivial. An affirmative answer would support their interpretation in terms of noncommutative Minkowski space. We hope to return to this problem elsewhere.

\section{Conclusions}

In this investigation we have clarified the relation between the warped convolution of $C^{*}$-dynamical systems, proposed in [13], and the strict deformation of such systems, established by Rieffel [28]. It turned out that, for fixed deformation matrix $Q$, the warped convolution induces a faithful covariant representation of the corresponding Rieffel algebra, if the original dynamical system is given in a faithful covariant representation. Thus, from this point of view, the warped convolution provides little new information. Yet, whereas the Rieffel deformations were introduced for the purpose of quantizing classical systems with Poisson bracket given by a fixed $Q$, warped convolutions were conceived for the deformation of quantum field theories. Within the latter framework one must deal simultaneously with a multitude of different deformation matrices $Q$ and establish relations between the resulting operators. The warping procedure is more appropriate in this context, since all warped deformations of a given dynamical system are concretely presented in a single Hilbert space, irrespective of the choice of $Q$.

For the discussion of the field theoretic aspects it has proven to be convenient to make use of the concept of causal Borchers triples $(\mathcal{R}, U, \Omega)$. The algebras of observables attached to arbitrary regions in Minkowski space can be reconstructed from any such triple, thereby specifying a covariant and causal quantum theory. Within this setting the problem of constructing a theory thus presents itself as follows. One first has to devise a continuous unitary representation $U$ of the Poincare group on some Hilbert 
space which satisfies the relativistic spectrum condition with vacuum vector $\Omega$. This task can be accomplished, e.g., by specifying the stable particle content of the theory and performing the standard Fock space construction. In a second step one must exhibit a von Neumann algebra $\mathcal{R}$ on this space satisfying certain specific compatibility conditions with respect to the action of $U$, which allow one to interpret $\mathcal{R}$ as an algebra of observables localized in a given wedge region of Minkowski space. It should be noted that the nets of local observable algebras appearing in any quantum field theory can be realized in this way.

Disregarding systems with an unreasonably large number of local degrees of freedom, the algebraic structure of $\mathcal{R}$ is known to be model independent, i.e. the algebras corresponding to different theories are isomorphic [6]. One may thus take as prototype the von Neumann algebra $\mathcal{R}_{0}$ generated by free (non-interacting) fields on Fock space which are smeared with test functions having support in the given wedge region. Despite this concrete setting, the problem of identifying other proper examples of such algebras $\mathcal{R}$ is notoriously difficult. The strategy pursued in the present investigation is based on the general idea of deforming a given causal Borchers triple, such as $\left(\mathcal{R}_{0}, U, \Omega\right)$, without changing the representation $U$. The warping procedure provides a consistent method to that effect. It leads to a family of new examples of causal Borchers triples in any number of spacetime dimensions.

However, the deformations of Borchers triples obtained by the warping procedure are rather special and of limited physical interest. It therefore seems worthwhile to fathom the potential of the general idea underlying this construction. Since the representation $U$ of $\mathcal{P}_{+}^{\uparrow}$ induces the pertinent constraints on the admissible algebras $\mathcal{R}$, one may try to generalize the formula for the warped deformations by the ansatz

$$
A \doteq \iint d \lambda d \lambda^{\prime} K\left(\lambda, \lambda^{\prime}\right) \alpha_{\lambda \lambda^{\prime}}\left(A_{0}\right) L\left(\lambda, \lambda^{\prime}\right), \quad A_{0} \in \mathcal{R}_{0},
$$

where $d \lambda$ denotes the Haar measure on $\mathcal{P}_{+}^{\uparrow}$ (or a subgroup thereof) and $K, L$ are suitable operator valued kernels. The consistency conditions on the algebra $\mathcal{R}$ generated by the deformed operators can then be re-expressed in terms of transformation properties of these kernels under the adjoint action of the representation $U$.

In two spacetime dimensions these constraints simplify considerably. There it suffices if the kernels $K, L$ transform covariantly under the adjoint action of the unitary representation $U$ of $\mathcal{P}_{+}^{\uparrow}$ and $\Omega$ is cyclic and separating for the resulting deformed von Neumann algebra $\mathcal{R}$. One may then proceed as in Sect. 3 and extend the representation $U$ to a representation of $\mathcal{P}_{+}$by adding to it the modular conjugation associated with $(\mathcal{R}, \Omega)$ which can be interpreted as PCT-operator. The algebras corresponding to arbitrary wedges can be obtained from $\mathcal{R}$ by the adjoint action of the resulting (anti)unitary representation of $\mathcal{P}_{+}$. Indeed, there is evidence that a large family of integrable models on two-dimensional Minkowski space, considered by one of us, can be subsumed in this manner $[24,25]$.

The prospect of finding other interesting deformations of this kind also in higher spacetime dimensions seems promising. Moreover, the method can also be transferred to quantum field theories on curved spacetimes having a sufficiently large isometry group [14]. Thus the algebraic methods presented here shed new light on the yet unsolved constructive problems in relativistic quantum field theory. 
Open Access This article is distributed under the terms of the Creative Commons Attribution Noncommercial License which permits any noncommercial use, distribution, and reproduction in any medium, provided the original author(s) and source are credited.

\section{Appendix}

We give here the proof of Lemma 4.6, which concludes that, given any neighborhood $\mathcal{N}$ of the identity in $\mathcal{P}_{+}^{\uparrow}, \Omega$ is cyclic for at most one of the algebras $\bigcap_{\lambda \in \mathcal{N}} \alpha_{\lambda}(\mathcal{R})$ and $\bigcap_{\lambda \in \mathcal{N}} \alpha_{\lambda}\left(\mathcal{R}_{Q}\right)$. Moreover, we comment on the significance of the spectral constraint made in the hypothesis of the lemma.

We begin by noting that it suffices to establish the assertion for arbitrarily small neighborhoods $\mathcal{N}$ of the identity in $\mathcal{P}_{+}^{\uparrow}$; for it then holds for all bigger neighborhoods as well. In particular, one may assume that $\lambda_{0} \mathcal{N} \lambda_{0}^{-1}=\mathcal{N}$, where $\lambda_{0} \in \mathcal{P}_{+}^{\uparrow}$ is a rotation by $\pi$ which maps $\mathcal{W}$ onto $\mathcal{W}^{\prime}$. Assume now that $\Omega$ is cyclic for $\mathcal{S} \doteq \bigcap_{\lambda \in \mathcal{N}} \alpha_{\lambda}(\mathcal{R}) \subset \mathcal{R}$ and let $A \in \mathcal{S} \cap \mathcal{C}^{\infty}$. Then for any $\lambda \in \mathcal{N}$ one has $\alpha_{\lambda-1}(A) \in \mathcal{R}^{\infty}$, so the warped operators $\alpha_{\lambda^{-1}}(A)_{Q}$ are well-defined and $\alpha_{\lambda}\left(\alpha_{\lambda^{-1}}(A)_{Q}\right) \in \alpha_{\lambda}\left(\mathcal{R}_{Q}\right)$. By Proposition 2.9 $\alpha_{\lambda}\left(\alpha_{\lambda^{-1}}(A)_{Q}\right)=A_{\Lambda Q \Lambda^{T}}$, where $\Lambda$ is the image of $\lambda$ under the canonical homomorphism mapping $\mathcal{P}_{+}^{\uparrow}$ onto $\mathcal{L}_{+}^{\uparrow}$. Hence $A_{\Lambda Q \Lambda^{T}} \in \alpha_{\lambda}\left(\mathcal{R}_{Q}\right), \lambda \in \mathcal{N}$.

Assume now, for a reductio ad absurdum, that $\Omega$ is also cyclic for $\bigcap_{\lambda \in \mathcal{N}} \alpha_{\lambda}\left(\mathcal{R}_{Q}\right)$. Then, since

$$
\left(\bigvee_{\lambda \in \mathcal{N}} \alpha_{\lambda}\left(\mathcal{R}_{Q}\right)\right)^{\prime}=\bigcap_{\lambda \in \mathcal{N}} \alpha_{\lambda}\left(\mathcal{R}_{Q}^{\prime}\right) \supset \bigcap_{\lambda \in \mathcal{N}} \alpha_{\lambda}\left(\alpha_{\lambda_{0}}\left(\mathcal{R}_{Q}\right)\right)=\alpha_{\lambda_{0}}\left(\bigcap_{\lambda \in \mathcal{N}_{0}} \alpha_{\lambda}\left(\mathcal{R}_{Q}\right)\right)
$$

where the inclusion obtains because $\left(\mathcal{R}_{Q}, U, \Omega\right)$ is a causal Borchers triple, one concludes that $\Omega$ is separating for $\bigvee_{\lambda \in \mathcal{N}} \alpha_{\lambda}\left(\mathcal{R}_{Q}\right)$. But Eq. (2.7) entails $A_{\Lambda Q \Lambda^{T}} \Omega=A_{Q} \Omega$, and consequently $A_{\Lambda Q \Lambda^{T}}=A_{Q}, \lambda \in \mathcal{N}$. Proposition 2.11 then yields $A_{\Lambda Q \Lambda^{T}-Q}=$ $A=A_{Q-\Lambda Q \Lambda^{T}}, \lambda \in \mathcal{N}$. By explicit computation one finds that the sums of matrices of the form $\Lambda Q \Lambda^{T}-Q, \lambda \in \mathcal{N}$, include all multiples of $Q$. Hence $A_{m} Q=A, m \in \mathbb{Z}$, by another application of Proposition 2.11. The same is true for the smooth operators $A^{\prime} \in \mathcal{T} \doteq \alpha_{\lambda_{0}}(\mathcal{S}) \subset \alpha_{\lambda_{0}}(\mathcal{R}) \subset \mathcal{R}^{\prime}$, as one sees by applying again Proposition 2.9.

Pick now an arbitrary compact subset $\Gamma$ in the interior of the forward lightcone $V_{+}$, so $Q \Gamma$ is a compact subset in the interior of $\mathcal{W}$. Hence for any given $x, y \in \mathbb{R}^{n}$ and sufficiently large $m \in \mathbb{N}$, the wedges $\mathcal{W}+x+m Q u$ and $\mathcal{W}^{\prime}+y-m Q v$ lie spacelike to each other for all $u \in \Gamma$ and $v \in V_{+}$. As explained in Sect. 4, one therefore has for any $A \in \mathcal{R}, A^{\prime} \in \mathcal{R}^{\prime}$, the equality $\left[\alpha_{x+m} Q u(A), \alpha_{y-m} Q v\left(A^{\prime}\right)\right]=0$. Now let $A \in \mathcal{S} \cap \mathcal{C}^{\infty}$, $A^{\prime} \in \mathcal{T} \cap \mathcal{C}^{\infty}$, let $\Phi$ be any vector with spectral support with respect to $U \uparrow \mathbb{R}^{n}$ contained in $\Gamma$, and let $\Psi$ be any other vector with compact spectral support. According to the preceding step and Proposition 2.9 one has $\alpha_{x}(A)=\alpha_{x}\left(A_{m Q}\right)=\left(\alpha_{x}(A)\right)_{m Q}$ and similarly $\alpha_{y}\left(A^{\prime}\right)=\left(\alpha_{y}\left(A^{\prime}\right)\right)_{-m Q}$. So one obtains by the same line of arguments as in the proof of Proposition 2.10,

$$
\begin{aligned}
\left\langle\Phi, \alpha_{x}(A) \alpha_{y}\left(A^{\prime}\right) \Psi\right\rangle & =\lim _{m \rightarrow \infty}\left\langle\Phi,\left(\alpha_{x}(A)\right)_{m Q}\left(\alpha_{y}\left(A^{\prime}\right)\right)_{-m Q} \Psi\right\rangle \\
& =\lim _{m \rightarrow \infty}\left\langle\Phi,\left(\alpha_{y}\left(A^{\prime}\right)\right)_{-m Q}\left(\alpha_{x}(A)\right)_{m Q} \Psi\right\rangle \\
& =\left\langle\Phi, \alpha_{y}\left(A^{\prime}\right) \alpha_{x}(A) \Psi\right\rangle,
\end{aligned}
$$


where in the second equality the support properties of $\Phi$ and the above commutation properties of $A, A^{\prime}$ have been used. Thus, varying $\Phi, \Psi$ within the above limitations, one arrives at

$$
E(\Gamma)\left[\alpha_{x}(A), \alpha_{y}\left(A^{\prime}\right)\right]=0 \text { for } x, y \in \mathbb{R}^{n},
$$

where $E(\cdot)$ denotes the spectral resolution of $U \uparrow \mathbb{R}^{n}$.

This equality has been established for $A \in \mathcal{S} \cap \mathcal{C}^{\infty}$ and $A^{\prime} \in \mathcal{T} \cap \mathcal{C}^{\infty}$. But if $\mathcal{N}$ is sufficiently small, the algebra $\mathcal{S}$ is mapped into itself by all translations in the open convex cone $\bigcap_{\lambda \in \mathcal{N}} \Lambda \mathcal{W}$; appealing to the discussion following relation (3.1) allows one to conclude that $\mathcal{S} \cap \mathcal{C}^{\infty}$ is weakly dense in $\mathcal{S}$ and, similarly, $\mathcal{T} \cap \mathcal{C}^{\infty}$ is weakly dense in $\mathcal{T}$. So the equality holds for all $A \in \mathcal{S}$ and $A^{\prime} \in \mathcal{T}$. Moreover, for any $u, v \in \mathbb{R}^{n}$ there is a $w \in \mathbb{R}^{n}$ such that $\alpha_{w}(\mathcal{T}) \supset \alpha_{u}(\mathcal{T}) \bigvee \alpha_{v}(\mathcal{T})$. (This follows from the the Poincaré covariance discussed in Sect. 4 and the geometry of wedge regions). Hence $E(\Gamma)[A, T]=0$ for $A \in \mathcal{S}$ and $T \in \bigvee_{y \in \mathbb{R}^{n}} \alpha_{y}(\mathcal{T})$.

Since $\Omega$ is cyclic for $\mathcal{S}$ it is also cyclic for $\mathcal{T}=\alpha_{\lambda_{0}}(\mathcal{S})$. The spectral condition on $U \uparrow \mathbb{R}^{n}$ therefore implies that the elements of $\bigcap_{y \in \mathbb{R}^{n}} \alpha_{y}(\mathcal{T})^{\prime}$ are invariant under translations. In particular $U(x) \in \bigvee_{y \in \mathbb{R}^{n}} \alpha_{y}(\mathcal{T}), x \in \mathbb{R}^{n}, c f$. [1, Theorem 4.6]. Thus $E(\Gamma)[A, U(x)]=0, x \in \mathbb{R}^{n}$, and consequently $E(\Gamma) A \Omega=0, A \in \mathcal{S}$. It is then clear that $E(\Gamma)=0$ for any compact subset $\Gamma$ in the interior of $V_{+}$. So the spectrum of $U \uparrow \mathbb{R}^{n}$ is confined to the boundary of the lightcone $V_{+}$, i.e. there is no spectral point in its interior, contradicting the hypothesis of the lemma. This completes the proof of the lemma.

Finally, let us discuss the significance of the assumption that the spectrum of $U \uparrow \mathbb{R}^{n}$ intersects the interior of $V_{+}$. As a matter of fact, disregarding the trivial case $\operatorname{sp} U=\{0\}$, this input is a consequence of the additivity of the energy-momentum spectrum, which can be established in the present setting if $\Omega$ is (apart from a phase) the only unit vector in the underlying Hilbert space which is invariant under translations [20, Chap. II.5.4]. The possibility that $\operatorname{sp} U$ consists of the boundary of $V_{+}$(and thus is not additive) can only be realized in theories where the Lorentz symmetry is spontaneously broken. With the help of one-dimensional chiral fields which one assigns to lightrays, one can manufacture such examples, and these are stable under the warping procedure. Since these examples seem to be merely of academic interest, we do not present them here.

\section{References}

1. Araki, H.: Mathematical Theory of Quantum Fields. Oxford: Oxford University Press, 1999

2. Baumgärtel, H., Wollenberg, M.: Causal Nets of Operator Algebras. Berlin: Akademie Verlag, 1992

3. Borchers, H.-J.: The CPT-theorem in two-dimensional theories of local observables. Commun. Math. Phys. 143, 15-332 (1992)

4. Borchers, H.-J.: On revolutionizing quantum field theory with Tomita's modular theory. J. Math. Phys. 41, 3604-3673 (2000)

5. Brunetti, R., Guido, D., Longo, R.: Modular localization and Wigner particles. Rev. Math. Phys. 14, 759785 (2002)

6. Buchholz, D., D’Antoni, C., Fredenhagen, K.: The universal structure of local algebras. Commun. Math. Phys. 111, 123 (1987)

7. Buchholz, D., Dreyer, O., Florig, M., Summers, S.J.: Geometric modular action and spacetime symmetry groups. Rev. Math. Phys. 12, 475-560 (2000)

8. Buchholz, D., Fredenhagen, K.: Locality and the structure of particle states. Commun. Math. Phys. 84, 1-54 (1982)

9. Buchholz, D., Lechner, G.: Modular nuclearity and localization. Ann. Henri Poincaré 5, 1065-1080 (2004)

10. Buchholz, D., Summers, S.J.: An Algebraic characterization of vacuum states in Minkowski space. 3. Reflection maps. Commun. Math. Phys. 246, 625-641 (2004) 
11. Buchholz, D., Summers, S.J.: Stable quantum systems in Anti-de Sitter space: Causality, independence and spectral properties. J. Math. Phys. 45, 4810-4831 (2004)

12. Buchholz, D., Summers, S.J.: String- and brane-localized causal fields in a strongly nonlocal model. J. Phys. A 40, 2147-2163 (2007)

13. Buchholz, D., Summers, S.J.: Warped convolutions: A novel tool in the construction of quantum field theories. In: Quantum Field Theory and Beyond, edited by Seiler, E., Sibold, K. Singapore: World Scientific, 2008, pp. 107-121

14. Dappiaggi, C., Lechner, G., Morfa-Morales, E.: Deformations of quantum field theories on spacetimes with Killing vector fields. Commun. Math. Phys. (2010). arXiv:1006.3548 (to appear)

15. Florig, M.: On Borchers' theorem. Lett. Math. Phys. 46, 289-293 (1998)

16. Glimm, J., Jaffe, A.: Quantum Physics. A Functional Integral Point of View, Berlin-Heidelberg-New York: Springer Verlag, 1987

17. Grosse, H., Lechner, G.: Wedge-local quantum fields and noncommutative Minkowski space. JHEP 0711, 012 (2007)

18. Grosse, H., Lechner, G.: Noncommutative deformations of Wightman quantum field theories. JHEP $\mathbf{0 8 0 9}$, $131(2008)$

19. Guido, D.: Modular covariance, PCT, Spin and Statistics. Ann. Inst. Henri Poincaré 63, 383-398 (1995)

20. Haag, R.: Local Quantum Physics. Berlin, Heidelberg and New York: Springer Verlag, 1992

21. Kaschek, D., Neumaier, N., Waldmann, S.: Complete positivity of Rieffel's quantization by actions of $\mathbb{R}^{d}$. J. Noncommut. Geom. 3, 361-375 (2009)

22. Lechner, G.: Polarization-free quantum fields and interaction. Lett. Math. Phys. 64, 137-154 (2003)

23. Lechner, G.: On the existence of local observables in theories with a factorizing S-matrix. J. Phys. A 38, 3045-3056 (2005)

24. Lechner, G.: Construction of quantum field theories with factorizing S-matrices. Commun. Math. Phys. 277, 821-860 (2008)

25. Lechner, G.: Article in preparation

26. Mund, J., Schroer, B., Yngvason, J.: String-localized quantum fields and modular localization. Commun. Math. Phys. 268, 621-672 (2006)

27. Pedersen, G.K.: $C^{*}$-Algebras and Their Automorphism Groups. London-New York-San Francisco: Academic Press, 1979

28. Rieffel, M.A.: Deformation quantization for actions of $\mathbb{R}^{d}$. Memoirs A.M.S. 506, 1-96 (1993)

29. Schroer, B.: Modular localization and the bootstrap-formfactor program. Nucl. Phys. B 499, 547568 (1997)

30. Takesaki, M.: Tomita's Theory of Modular Hilbert Algebras and Its Applications. Berlin-Heidelberg-New York: Springer Verlag, 1970

31. Takesaki, M.: Theory of Operator Algebras. Volume II, Berlin-Heidelberg-New York: Springer Verlag, 2003

Communicated by Y. Kawahigashi 